\title{
Accurate Impressive Optical Solitons For Nonlinear Refractive Index Cubic-Quartic through Birefringent Fibers
}

Emad H.M. Zahran

Benha University

Ahmet Bekir ( $\square$ bekirahmet@gmail.com )

Eskisehir https://orcid.org/0000-0001-9394-4681

\section{Research Article}

Keywords: The solitary wave ansatz method, the optical soliton for the nonlinear refractive index cubicquartic through birefringent fibers.

Posted Date: December 29th, 2021

Dol: https://doi.org/10.21203/rs.3.rs-1026889/v1

License: (c) (i) This work is licensed under a Creative Commons Attribution 4.0 International License.

Read Full License 


\title{
Accurate impressive optical solitons for nonlinear refractive index cubic-quartic through birefringent fibers
}

\author{
Emad H.M. Zahran ${ }^{1}$, Ahmet Bekir ${ }^{2}$ \\ ${ }^{1}$ Departmentof Mathematical and Physical Engineering,BenhaUniversity,Faculty of \\ Engineering, Shubra, Egypt \\ ${ }^{2}$ Neighbourhood of Akcaglan, Imarli Street, Number: 28/4, 26030, Eskisehir, Turkey \\ E-mail: emad.zahran@feng.bu.edu.eg ; bekirahmet@gmail.com
}

\begin{abstract}
The main target of this work implements new accurate impressive optical solitons for four forms of the nonlinear refractive index cubic-quartic through birefringent fibers which play a vital role in all modern telecommunications process. These four different forms listed under whose profile names which are the cubic-quartic in polarization-preserving fibers with its different forms which are the kerr-law, the quadratic-law, the parabolic-law and the nonlocal-law NLSE. These new accurate optical solitons for these different forms are extracted in the framework of the solitary wave ansatz method (SWAM) which is powerful technique that achieves accurate results for all problems that are solved in the framework of it. The achieved results will be compared by the previous results which are achieved via other authors.
\end{abstract}

Keywords: The solitary wave ansatz method; the optical soliton for the nonlinear refractive index cubic-quartic through birefringent fibers.

\section{1-Introduction}

In a birefringent material, a wave consists of two polarization components which generally are governed by different effective refractive indices. This phenomenon has been observed in calcite, a crystal having one of the strongest birefringences via the Danish scientist Rasmus Bartholin [1]. Furthermore, through the nineteenth century Augustin-Jean Fresnel gives good representation of this phenomenon in terms of polarization and he can understand that the light can be considered as a wave with field components in transverse polarization. Much of the experiments related to polarization preceded the understanding of light as a transverse electromagnetic wave, and this has affected some terminology in use. Isotropic materials have symmetry in all directions and the refractive index is the same for any polarization direction. An anisotropic material is called "birefringent" because it will generally refract a single incoming ray in two directions, which we now understand correspond to the two different polarizations. This is true of either a uniaxial in which one ray behaves according to the normal law of refraction (corresponding to the ordinary refractive index) or biaxial material in which the polarization can deviate from normal incidence, which cannot be described using the law of refraction. The birefringence is usually obtained using an anisotropic crystal, it can be achieved from an optically isotropic material through few paths namely, Stress birefringence results when isotropic materials are stressed or deformed, Circular birefringence in liquids where there is an enantiomeric excess in a solution containing a molecule which has stereo isomers, via the Kerr effect, whereby an applied electric field induces birefringence at optical frequencies through the effect of nonlinear optics, via the Faraday effect, where a magnetic field causes some materials to become circularly birefringent. 
It is well known that both the nonlinear optical solitons dynamics and the Modelung-fluids are depending on the generalized nonlinear Schrödinger dispersive equation and resonant nonlinear Schrödinger dispersive equation. The propagation of solitons over intercontinental ranges depends on two principal key namely the dispersion and the nonlinearity, hence improvement of these two key will improve the transition of this waves. The four suggested models are derived from the well-known NLSE with dispersion term which describes the propagation of waves in optical fiber and has been perfectly examined for many physical problems. Recently, two trails to study the Birefringence for which the optical property of a material having a refractive index that depends on the polarization and distribution direction of light via [2,3]. Moreover, some studies through many authors are established to study various forms of NLSE which describes different models which arising in nonlinear phenomenon [4-14]. Furthermore recent studies for cubic-quartic to achieve the optical solitons are proposed through [15-27]. Specially, few modern preliminary results to study the nonlinear refractive index cubic-quartic through birefringent fibers have been reported during [28-31].

The main purpose of this article is establishing new impressive perceptions of the emerged solitons to the four forms of the nonlinear refractive index cubic-quartic through birefringent fibers which are kerr-law, parabolic law, quadratic-law and non-local law nonlinearity in the framework of the SWAM [25-26], [31-32].

The proposed method is one of the famous ansatz methods which doesn't surrender to the balance rule, achieve analytical solutions and it examined previously for many NLPDE. This method is first suggested by Biswas [31], Triki and Wazwaz [32] who successes to find the exact bright and dark soliton solutions to the perturbed nonlinear Schrödinger equation with the Kerr law nonlinearity and the higher-order nonlinear Schrödinger equation.

\section{Description of the SWAM}

To discuss the discribtion of this method let us overview about the nonlinear evolution equation which can be written in the form,

$$
G\left(u, u_{x}, u_{t}, u_{x x}, u_{t t}, \ldots \ldots\right)=0 .
$$

Where $G$ is a function of $u(x, t)$ and its partial derivatives that involves the highest order derivatives and nonlinear terms, according to the transformation $u(x, t)=u(\zeta), \zeta=x-C_{0} t$ equation (1) can be reduced to the following ODE:

$$
H\left(u, u^{\prime}, u^{\prime \prime}, u^{\prime \prime \prime} \ldots \ldots \ldots . . . .\right)=0
$$

wher $H$ is function in $u(\zeta)$ and its total derivatives, while ${ }^{\prime}=\frac{d}{d \zeta}$.

We can admits the solution forms of the SWAM from the point of view of [23-24], [30-32] as follow, let us assume the transformation,

$$
u(x, t)=\psi(x, t) e^{i R(x, t)}
$$

Where $\psi(x, t)$ and $R(x, t)$ are the portion amplitude and phase portion of the soliton respectively. Equation (3) with simple calculations will extract the following relations,

$$
\begin{aligned}
& u_{t}=\left(\psi_{t}+i \psi R_{t}\right) e^{i R} \\
& u_{x}=\left(\psi_{x}+i \psi R_{x}\right) e^{i R} \\
& u_{x x}=\left(\psi_{x x}+2 i \psi_{x} R_{x}+i \psi R_{x x}-\psi R_{x}^{2}\right) e^{i R}
\end{aligned}
$$




$$
\begin{aligned}
& u_{x x x}=\left(\psi_{x x x}+3 i R_{x} \psi_{x x}+3 i R_{x x} \psi_{x}+i R_{x x x} \psi-i R_{x}^{3} \psi-3 R_{x} R_{x x} \psi\right) e^{i R} \\
& u_{x x x}=\left(\psi_{x x x}+4 i R_{x} \psi_{x x}-3 R_{x}^{2} \psi_{x x}-6 R_{x} R_{x x} \psi_{x}+3 i R_{x x} \psi_{x x}-R R_{x x} \psi+i R_{x x} \psi_{x}+R_{x}^{4} \psi-i R_{x}^{3} \psi_{x}-3 i R_{x}^{2} R_{x x} \psi\right) e^{i R}
\end{aligned}
$$

Now we will investigate the bright and dark soliton solutions individually as follows,

\section{The bright soliton solutions}

$$
\begin{aligned}
& \psi(x, t)=A_{1} \operatorname{sech}^{R_{1}} t_{1}, \text { where } t_{1}=B\left(x-w_{1} t\right) \text { and } R_{1}(x, t)=k x-\Omega t \\
& \psi_{t}=-A_{1} B w_{1} R_{1} \operatorname{sech}^{R_{1}} t_{1} \tanh t_{1} \\
& \psi_{x}=A_{1} B R_{1} \operatorname{sech}^{R_{1}} t_{1} \tanh t_{1} \\
& \psi_{x x}=A_{1} B^{2} R_{1}\left(1+R_{1}\right) \operatorname{sech}^{R_{1}+2} t_{1}-A_{1} B^{2} R_{1}^{2} \operatorname{sech}^{R_{1}} t_{1} \\
& \psi_{x x x}=A_{1} B^{3} R_{1}\left(R_{1}+1\right)\left(R_{1}+2\right) \operatorname{sech}^{R_{1}+2} t_{1} \tanh t_{1}-A_{1} B^{3} R_{1}^{3} \operatorname{sech}^{R_{1}} t_{1} \tanh t_{1} \\
& \psi_{x x x}=A B^{3} R_{1}\left(R_{1}+1\right)\left(R_{1}+2\right)^{2} \operatorname{sech}^{R_{1}+2} t_{1}-A_{1} B^{3} R_{1}\left(R_{1}+1\right)^{2}\left(R_{1}+2\right) \operatorname{sech}^{R_{1}+4} t_{1}-A_{1} B^{3} R_{1}^{4} \operatorname{sech}^{R_{1}} t_{1}
\end{aligned}
$$

\section{The dark soliton solutions}

$$
\begin{aligned}
& \psi(x, t)=A_{2} \tanh ^{R_{2}} t_{2}, \text { where } t_{2}=B\left(x-w_{2} t\right) \text { and } R_{2}(x, t)=k x-\Omega t \\
& \psi_{t}=-A_{2} B R_{2} w_{2}\left[\tanh ^{R_{2}-1} t_{2}-\tanh ^{R_{2}+1} t_{2}\right] \\
& \psi_{x}=A_{2} B R_{2}\left[\tanh ^{R_{2}-1} t_{2}-\tanh ^{R_{2}+1} t_{2}\right] \\
& \psi_{x x}=A_{2} R_{2}\left(R_{2}-1\right) B^{2} \tanh ^{R_{2}-2} t_{2}-2 A_{2} R_{2}^{2} B^{2} \tanh ^{R_{2}} t_{2}+ \\
& \quad A_{2} R_{2}\left(R_{2}+1\right) B^{2} \tanh ^{R_{2}+2} t_{2} \\
& \psi_{x x x}=A_{2} B^{3} R_{2}\left(R_{2}-1\right)\left(R_{2}-2\right) \tanh ^{R_{2}-3} t_{2}-\left[A_{2} B^{3} R_{2}\left(R_{2}-1\right)\left(R_{2}-2\right)+2 A_{2} R_{2}^{3} B^{3}\right] \tanh ^{R_{2}-1} t_{2}+ \\
& \quad\left[A_{2} B^{3} R_{2}\left(R_{2}+1\right)\left(R_{2}+2\right)+2 A_{2} R_{2}^{3} B^{3}\right] \tanh ^{R_{2}+1} t_{2}-A_{2} B^{3} R_{2}\left(R_{2}+1\right)\left(R_{2}+2\right) \tanh ^{R_{2}+3} t_{2} \\
& \psi_{x x x x}=A_{2} B^{3} R_{2}\left(R_{2}+1\right)\left(R_{2}+2\right)\left(R_{2}+3\right) \tanh ^{R_{2}+4} t_{2}+A_{2} B^{3} R_{2}\left(R_{2}-1\right)\left(R_{2}-2\right)\left(R_{2}-3\right) \tanh ^{R_{2}-4} t_{2} \\
& \quad+2 A_{2} B^{3} R_{2}\left(R_{2}+1\right)^{2}\left(R_{2}-2\right) \tanh ^{R_{2}+2} t_{2}-A_{2} B^{3} R_{2}\left(R_{2}-1\right)\left(3 R_{2}^{2}+8 R_{2}+8\right) \tanh ^{R_{2}-2} t_{2} \\
&- A_{2} B^{3} R_{2}\left(R_{2}^{4}+4 R_{2}^{3}+17 R_{2}^{2}+2 R_{2}+4\right) \tanh ^{R_{2}} t_{2}
\end{aligned}
$$

\section{Application of the SWAM to the cubic-quartic for the kerr-law NLSE in polarization-preserving fibers}

\subsection{The bright cubic-quartic solitons}

In the framework of [28-29] the cubic-quartic for the kerr-law NLSE in polarizationpreserving fibers can be proposed in the form,

$$
i Q_{t}+i a Q_{x x x}+b Q_{x x x x}+c\left|Q^{2 m}\right| Q=0
$$

Where $a, b$ and $c$ denote respectively to the 3 -th order dispersion index, the 4-th order dispersion index, kerr-law refractive coefficient while $i=\sqrt{-1}$.

The cubic-quartic NLSE in birefringent fiber for kerr-law are,

$$
\begin{aligned}
& i u_{t}+i a_{1} u_{x x x}+b_{1} u_{x x x x}+\left[c_{1}|u|^{2}+d_{1}|v|^{2}\right] u=0 \\
& i v_{t}+i a_{2} v_{x x x}+b_{2} v_{x x x x}+\left[c_{2}|v|^{2}+d_{2}|u|^{2}\right] v=0
\end{aligned}
$$

Via substituting the relations (3-8) into any one of Eq. (22) mentioned above we obtain,

$$
\begin{aligned}
& i\left(\psi_{t}-i \Omega \psi\right) e^{i R_{1}}+i a_{1}\left(\psi_{x x x}+3 i k \psi_{x x}-i k^{3} \psi\right) e^{i R_{1}} \\
& +b_{1}\left(\psi_{x x x x}+4 i k \psi_{x x x}-3 k^{2} \psi_{x x}+k^{4} \psi-i k^{3} \psi\right) e^{i R_{1}}+\left(c_{1}+d_{1}\right) \psi^{3} e^{i R_{1}}=0
\end{aligned}
$$


From which the following real and imaginary parts are respectively emerged:

$$
\begin{aligned}
& \operatorname{Re} \Rightarrow b_{1} \psi_{x x x x}-3 k\left(a_{1}+k\right) \psi_{x x}+\left(\Omega+k^{3}+k^{4}\right) \psi+\left(c_{1}+d_{1}\right) \psi^{3}=0 \\
& \operatorname{Im} \Rightarrow \psi_{t}+\left(a_{1}+4 b_{1} k\right) \psi_{x x x}-b_{1} k^{3} \psi_{x}=0
\end{aligned}
$$

Via inserting the relations (9-14) at the real and imaginary parts equations (24), (25) we get,

$$
\begin{aligned}
& {\left[b_{1} A_{1} B^{3} R_{1}\left(R_{1}+1\right)\left(R_{1}+2\right)-3 k\left(a_{1}+k\right) A_{1} B^{2} R_{1}\left(1+R_{1}\right)\right] \operatorname{sech}^{R_{1}+2} t_{1}} \\
& +\left[3 k\left(a_{1}+k\right) A_{1} B^{2} R_{1}^{2}+A_{1}\left(\Omega+k^{3}+k^{4}\right)-A_{1} B^{3} R_{1}^{4}\right] \operatorname{sech}^{R_{1}} t_{1} \\
& -A_{1} B^{3} R_{1}\left(R_{1}+1\right)^{2}\left(R_{1}+2\right) \operatorname{sech}^{R_{1}+4} t_{1}+\left(c_{1}+d_{1}\right) A_{1}^{3} \operatorname{sech}^{3 R_{1}} t_{1}=0 \\
& \left(a_{1}+4 k b_{1}\right) A_{1} B^{3} R_{1}\left(R_{1}+1\right)\left(R_{1}+2\right) \operatorname{sech}^{R_{1}+2} t_{1} \tanh t_{1}-\left(a_{1}+4 k b_{1}\right) A_{1} B^{3} R_{1}^{3} \operatorname{sech}^{R_{1}} t_{1} \tanh t_{1}- \\
& b_{1} A_{1} R_{1} k^{3} B \operatorname{sech}^{R_{1}} t_{1} \tanh t_{1}-A_{1} B w_{1} R_{1} \operatorname{sech}^{R_{1}} t_{1} \tanh t_{1}=0 .
\end{aligned}
$$

Equation (26) implies $R_{1}=2$, when the equivalence between the higher orders of $\operatorname{sech}^{i} t_{1}$ is achieved the following relations will be extracted $A_{1}^{2}=\frac{72 B^{3}}{c_{1}+d_{1}}, \quad B=\frac{3 k\left(a_{1}+k\right)}{4 b_{1}}$ and $\Omega=16 B^{3}-k^{4}-k^{3}-12 k\left(a_{1}+k\right) B^{2}$, moreover the imaginary part implies $a_{1}=-4 b_{1} k, w_{1}=b_{1} k^{3}$. From the above relations we get $A_{1}= \pm 114.6 i, a_{1}=-4, b_{1}=c_{1}=d_{1}=w_{1}=1, B=-4.5, \Omega=-729$ Now, in the framework of these obtained constants and the suggested method the solution is:

$$
\begin{aligned}
& u= \pm 114.6 i \operatorname{sech}^{2}[-4.5(x-t)] e^{i(x+729 t)} \\
& \operatorname{Re} u= \pm 114.6 \operatorname{sech}^{2}[-4.5(x-t)] \times \sin (x+729 t) \\
& \operatorname{Im} u= \pm 114.6 \operatorname{sech}^{2}[-4.5(x-t)] \times \cos (x+729 t)
\end{aligned}
$$

For simplicity we will plot only one for each case say the positive one for each case,

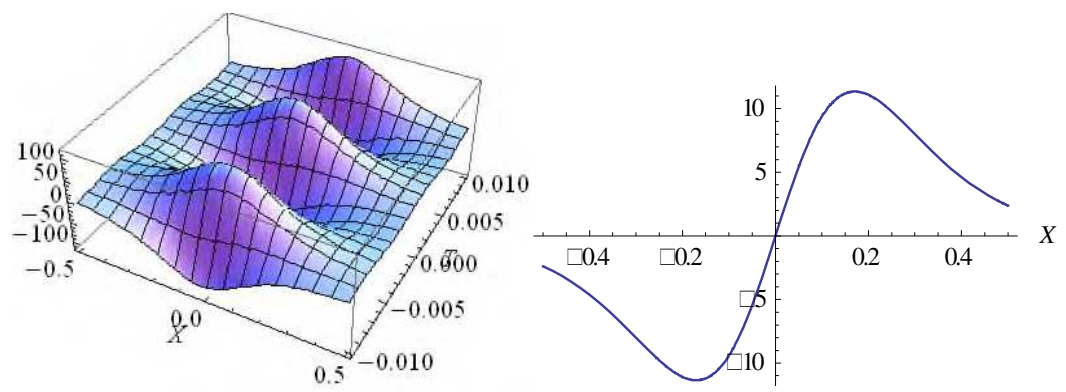

Fig. 1 : The bright cubic-quartic soliton for kerr law the Re. part Eq.(29) in 2D and 3D with values:
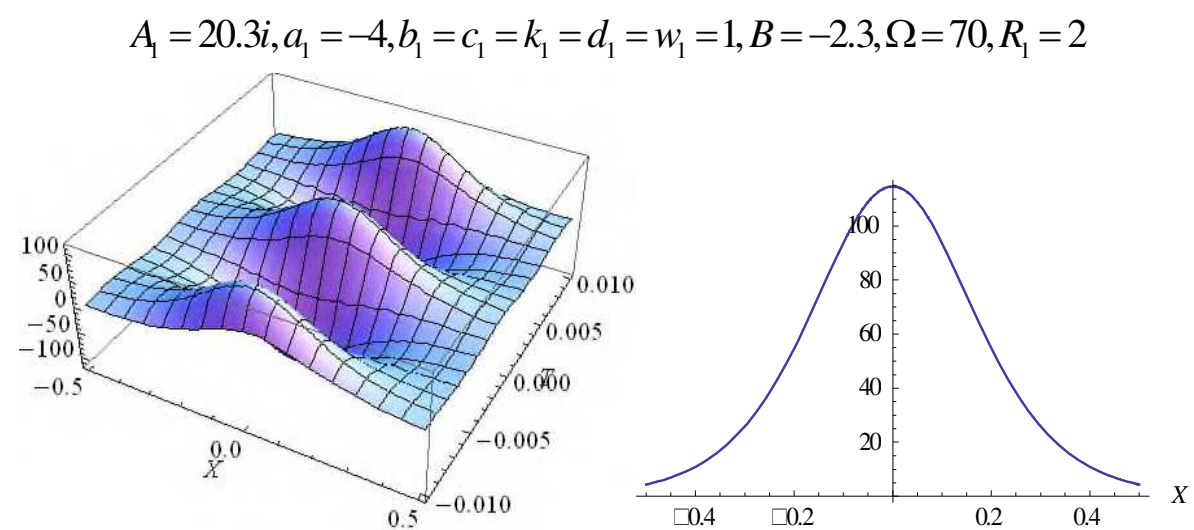

Fig. 2 : The bright cubic-quartic soliton for kerr law the Im. part Eq.(30) in 2D and 3D with values:

$$
A_{1}=20.3 i, a_{1}=-4, b_{1}=c_{1}=k_{1}=d_{1}=w_{1}=1, B=-2.3, \Omega=70, R_{1}=2
$$




\subsection{The dark cubic-quartic solitons}

Via inserting the relations (15-20) into the real and imaginary parts equations (24), (25) we get,

$$
\begin{aligned}
& b_{1} A_{2} B^{3} R_{2}\left(R_{2}+1\right)\left(R_{2}+2\right)\left(R_{2}+3\right) \tanh ^{R_{2}+4}\left(t_{2}\right)+b_{1} A_{2} B^{3} R_{2}\left(R_{2}-1\right)\left(R_{2}-2\right)\left(R_{2}-3\right) \tanh ^{R_{2}-4} t_{2} \\
& -\left[b_{1} A_{2} B^{3} R_{2}\left(R_{2}-1\right)\left(3 R_{2}^{2}+8 R_{2}+8\right)+3 k\left(a_{1}+k\right) A_{2} R_{2}\left(R_{2}-1\right) B^{2}\right] \tanh ^{R_{2}-2} t_{2} \\
& +\left[2 b_{1} A_{2} B^{3} R_{2}\left(R_{2}+1\right)^{2}\left(R_{2}-2\right)-3 k\left(a_{1}+k\right) A_{2} R_{2}\left(R_{2}+1\right) B^{2}\right] \tanh ^{R_{2}+2} t_{2}+ \\
& {\left[\left(\Omega+k^{3}+k^{4}\right) A_{2}+6 k\left(a_{1}+k\right) A_{2} R_{2}^{2} B^{2}-b_{1} A_{2} B^{3} R_{2}\left(R_{2}^{4}+4 R_{2}^{3}+17 R_{2}^{2}+2 R_{2}+4\right)\right] \tanh ^{R_{2}} t_{2}} \\
& +\left(c_{1}+d_{1}\right) A_{2}^{3} \tanh ^{3 R_{2}} t_{2}=0, \\
& {\left[A_{2} B R_{2} w_{2}+b_{1} k^{3} B A_{2} R_{2}\right] \tanh ^{R_{2}+1} t_{2}-\left[A_{2} B R_{2} w_{2}+b_{1} k^{3} B A_{2} R_{2}\right] \tanh ^{R_{2}-1} t_{2}} \\
& +\left(a_{1}+4 b_{1} k\right) A_{2} R_{2}\left(R_{2}+1\right) B^{2} \tanh ^{R_{2}+2} t_{2}+\left(a_{1}+4 b_{1} k\right) A_{2} R_{2}\left(R_{2}-1\right) B^{2} \tanh ^{R_{2}-2} t_{2} \\
& -\left(a_{1}+4 b_{1} k\right) 2 A_{2} R_{2}^{2} B^{2} \tanh ^{R_{2}} t_{2}=0
\end{aligned}
$$

When the equivalence between the higher orders of $\tanh ^{i} t_{2}$ is implemented for Eq.(31) the following relations $R_{2}=2, A_{2}^{2}=\frac{60 b_{1} B^{3}}{c_{1}+d_{1}}, \Omega+k^{3}+k^{4}=248 b_{1} B^{3}, a_{1}=-k$ will be achieved.

Moreover, equation (32) implies $b_{1}=0.25, w_{2}=-0.25, \Omega=26$ after choosing $B=1$, hence we can get these values, $A_{2}= \pm 3.9 i, a_{1}=-1, b_{1}=0.25, B=k_{1}=c_{1}=d_{1}=1, \Omega=26, w_{2}=-0.25, R_{2}=2$.

This result will generate two sub- results according to the value of $A_{2}$ which are,

$$
\begin{aligned}
& A_{2}=3.9 i, a_{1}=-1, b_{1}=0.25, B=k_{1}=c_{1}=d_{1}=1, \Omega=26, w_{2}=-0.25, R_{2}=2 \\
& A_{2}=-3.9 i, a_{1}=-1, b_{1}=0.25, B=k_{1}=c_{1}=d_{1}=1, \Omega=26, w_{2}=-0.25, R_{2}=2
\end{aligned}
$$

Now, for these two results the solutions in the framework of the proposed method and these obtained constants are:

$$
\begin{aligned}
& u(x, t)=A_{2} e^{i R_{2}(x, t)} \times \tanh ^{R_{2}} t_{2} \\
& u(x, t)= \pm 3.9 i e^{i(x-26 t)} \times \tanh ^{2}(x+0.25 t) \\
& \text { Re } u= \pm 3.9 \tanh ^{2}(x+0.25 t) \times \sin (x-26 t) \\
& \operatorname{Im} u= \pm 3.9 \tanh ^{2}(x+0.25 t) \times \cos (x-26 t)
\end{aligned}
$$

For simplicity we will plot only one for each case say the positive one for each case,
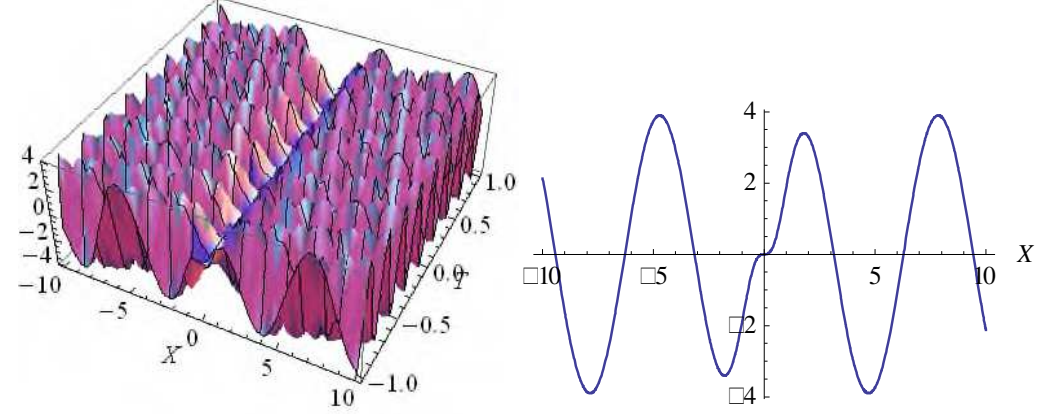

Fig. 3 : The dark cubic-quartic soliton for kerr law the Re. part Eq.(35) in 2D and 3D with values:

$$
A_{2}=3.9 i, a_{1}=-1, b_{1}=0.25, B=k_{1}=c_{1}=d_{1}=1, \Omega=26, w_{2}=-0.25, R_{2}=2
$$



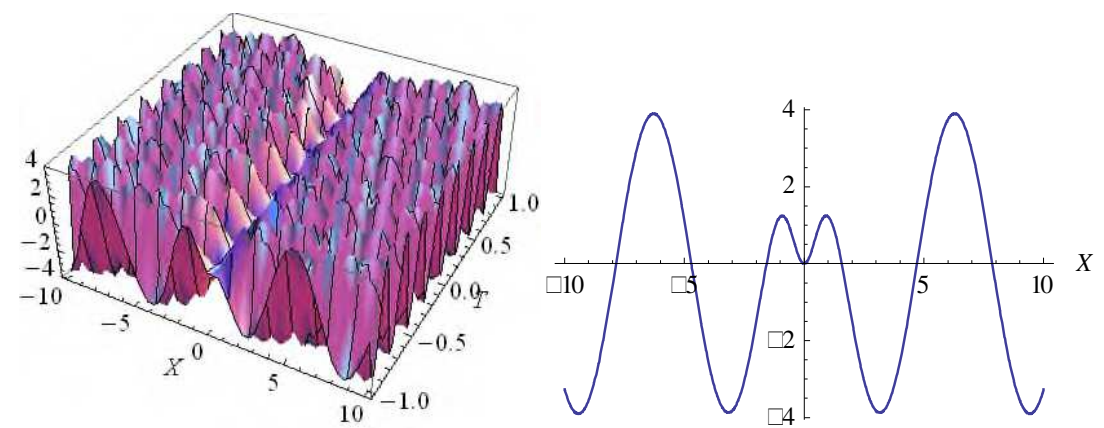

Fig. 4 : The bright cubic-quartic soliton for kerr law the Im. part Eq.(36) in 2D and 3D with values:

$$
A_{2}=3.9 i, a_{1}=-1, b_{1}=0.25, B=k_{1}=c_{1}=d_{1}=1, \Omega=26, w_{2}=-0.25, R_{2}=2
$$

\section{Application of the SWAM to the cubic-quartic for the Quadratic-Law NLSE in polarization-preserving fibers}

\subsection{The bright cubic-quartic solitons}

The cubic-quartic the NLSE in polarization-preserving fibers with quadratic- law can be proposed in the form,

$$
i Q_{t}+i a Q_{x x x}+b Q_{x x x x}=\left(c|Q|+d\left|Q^{2}\right|\right) Q
$$

The cubic-quartic NLSE in birefringent fiber for quadratic- law is,

$$
\begin{aligned}
& i u_{t}+i a_{1} u_{x x x}+b_{1} u_{x x x x}+c_{1} u \sqrt{|u|^{2}+|v|^{2}+u v^{*}+v u^{*}}+\left(d_{1}|u|^{2}+e_{1}|v|^{2}\right) u=0 \\
& i v_{t}+i a_{2} v_{x x x}+b_{2} v_{x x x}+c_{2} v \sqrt{|v|^{2}+|u|^{2}+u v^{*}+v u^{*}}+\left(d_{2}|v|^{2}+e_{2}|u|^{2}\right) v=0
\end{aligned}
$$

Via inserting the relations (3-8) into any one of the two equations (38) or (39) mentioned above we get,

$$
\begin{aligned}
& i\left(\psi_{t}-i \Omega \psi\right) e^{i R_{1}}+i a_{1}\left(\psi_{x x x}+3 i k \psi_{x x}-i k^{3} \psi\right) e^{i R_{1}}+b_{1}\left(\psi_{x x x}\right. \\
& \left.+4 i k \psi_{x x x}-3 k^{2} \psi_{x x}+k^{4} \psi-i k^{3} \psi\right) e^{i R_{1}}+2 c_{1} \psi^{3} e^{i R_{1}}+\left(e_{1}+d_{1}\right) \psi^{3} e^{i R_{1}}=0
\end{aligned}
$$

From which the following real and imaginary parts are respectively emerged as follow,

$$
\begin{aligned}
& \operatorname{Re} \Rightarrow b_{1} \psi_{x x x}-3 k\left(a_{1}+k\right) \psi_{x x}+\left(\Omega+k^{3}+k^{4}\right) \psi+2 c_{1} \psi^{2}+\left(e_{1}+d_{1}\right) \psi^{3}=0 \\
& \operatorname{Im} \Rightarrow \psi_{t}+\left(a_{1}+4 b_{1} k\right) \psi_{x x x}-b_{1} k^{3} \psi_{x}=0
\end{aligned}
$$

Via inserting the relations (9-14) into the real and imaginary parts equations (41), (42) respectively we get,

$$
\begin{aligned}
& {\left[b_{1} A_{1} B^{3} R_{1}\left(R_{1}+1\right)\left(R_{1}+2\right)-3 k\left(a_{1}+k\right) A_{1} B^{2} R_{1}\left(1+R_{1}\right)\right] \operatorname{sech}^{R_{1}+2} t_{1}} \\
& +\left[3 k\left(a_{1}+k\right) A_{1} B^{2} R_{1}^{2}+A_{1}\left(\Omega+k^{3}+k^{4}\right)-A_{1} B^{3} R_{1}^{4}\right] \operatorname{sech}^{R_{1}} t_{1}- \\
& A_{1} B^{3} R_{1}\left(R_{1}+1\right)^{2}\left(R_{1}+2\right) \operatorname{sech}^{R_{1}+4} t_{1}+2 c_{1} A_{1}^{2} \operatorname{sech}^{2 R_{1}} t_{1}+\left(e_{1}+d_{1}\right) A_{1}^{3} \operatorname{sech}^{3 R_{1}} t_{1}=0 \\
& \left(a_{1}+4 k b_{1}\right) A_{1} B^{3} R_{1}\left(R_{1}+1\right)\left(R_{1}+2\right) \operatorname{sech}^{R_{1}+2} t_{1} \tanh t_{1}-\left(a_{1}+4 k b_{1}\right) A_{1} B^{3} R_{1}^{3} \operatorname{sech}^{R_{1}} t_{1} \tanh _{1}- \\
& b_{1} A R_{1} k_{1}^{3} B \operatorname{sech}^{R_{1}} t_{1} \tanh t_{1}-A B w_{1} R_{1} \operatorname{sech}^{R_{1}} t_{1} \tanh t_{1}=0,
\end{aligned}
$$

When the equivalence between the higher orders of $\operatorname{sech}^{i} t_{1}$ is implemented at Eq. (43) the following results will be achieved, $R_{1}=2, A_{1}^{2}=\frac{72 B^{3}}{e_{1}+d_{1}}, B=\frac{4 k\left(a_{1}+k\right)}{4 b}$ and $\Omega=16 B^{3}-k^{4}-k^{3}-12 k\left(a_{1}+k\right) B^{2}$, 
and the imaginary part implies $a_{1}=-4 b_{1} k, w_{1}=b_{1} k^{3}$.

From which after choosing $b_{1}=c_{1}=d_{1}=e_{1}=1$ and solving the constructed system we obtain three solutions one of them is real and the other two solutions are imaginary, for simplicity we plot two of them which are

$$
\begin{aligned}
& \text { (1) } A_{1}=1.4, B=0.05, \Omega=-2.2, a_{1}=-4, b_{1}=c_{1}=d_{1}=w_{1}=1, R_{1}=2 \\
& \text { (2) } A_{1}=1.2-1.2 i, B=-2.3-0.3 i, \Omega=-7.9-201.7 i, a_{1}=-4, b_{1}=c_{1}=d_{1}=w_{1}=1, R_{1}=2
\end{aligned}
$$

For the first result the solution is,

$$
u=1.4 \operatorname{sech}^{2}[0.05(x-t)] e^{i(x+2.2 t)}
$$

$\operatorname{Re} u=1.4 \operatorname{sech}^{2}[0.05(x-t)] \times \cos (x+2.2 t)$

$\operatorname{Im} u=1.4 \operatorname{sech}^{2}[0.05(x-t)] \times \sin (x+2.2 t)$
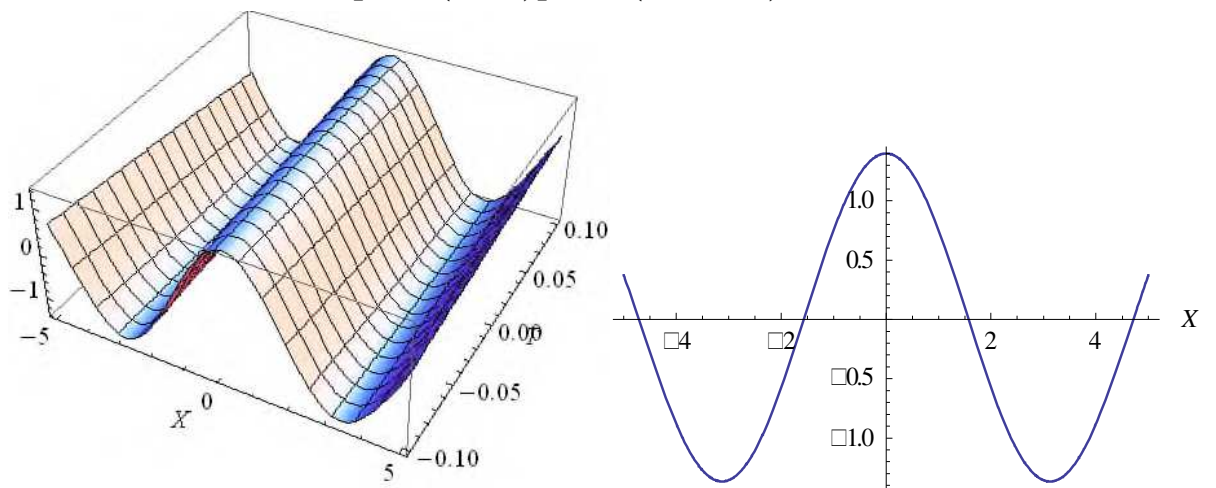

Fig. 5 : The bright cubic-quartic soliton for quadratic law the Re. part Eq.(47) in 2D and 3D with values: $A_{1}=1.4, B=0.05, \Omega=-2.2, a_{1}=-4, b_{1}=c_{1}=d_{1}=w_{1}=1, R_{1}=2$

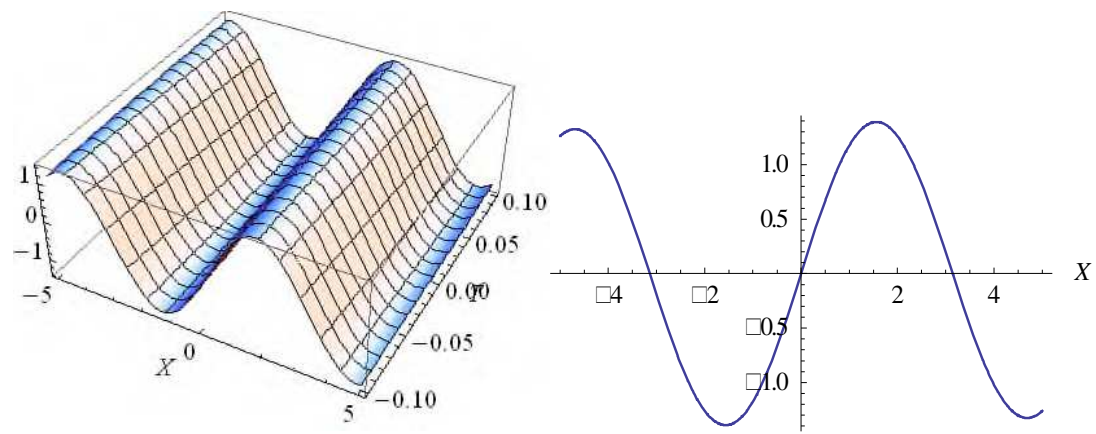

Fig. 6 : The bright cubic-quartic soliton for quadratic law the Im. part Eq.(48) in 2D and 3D with values: $A_{1}=1.4, B=0.05, \Omega=-2.2, a_{1}=-4, b_{1}=c_{1}=d_{1}=w_{1}=1, R_{1}=2$

The solution corresponding to the second result is,

$$
u=(1.2-1.2 i) \operatorname{sech}^{2}[(-2.3-0.3 i)(x-t)] e^{i[x-(7.9+201.7 i) t]}
$$


$\operatorname{Re} u=2 e^{201.7 t}\left(\frac{\left\{\begin{array}{l}(2.4 \cos [x-7.9 t]+2.4 \sin [x-7.9 t])\left(1+e^{4.6(x-t)}+e^{-4.6(x-t)}\right) \cos 0.6(x-t) \\ +(2.4 \sin [x-7.9 t]-2.4 \cos [x-7.9 t])\left(e^{4.6(x-t)}-e^{-4.6(x-t)}\right) \sin 0.6(x-t)\end{array}\right\}}{2+e^{9.2(x-t)}+e^{-9.2(x-t)}+\cos 1.2(x-t)}\right)$

$\operatorname{Im} u=2 e^{201.7 t}\left(\frac{\left\{\begin{array}{l}(2.4 \sin (x-7.9 t)-2.4 \cos (x-7.9 t))\left(1+\left[e^{4.6(x-t)}+e^{-4.6(x-t)}\right]\right) \cos 0.6(x-t) \\ -(2.4 \sin (x-7.9 t)+2.4 \cos (x-7.9 t))\left(e^{4.6(x-t)}-e^{-4.6(x-t)}\right) \sin 0.6(x-t)\end{array}\right\}}{2+e^{9.2(x-t)}+e^{-9.2(x-t)}+\cos 1.2(x-t)}\right)$
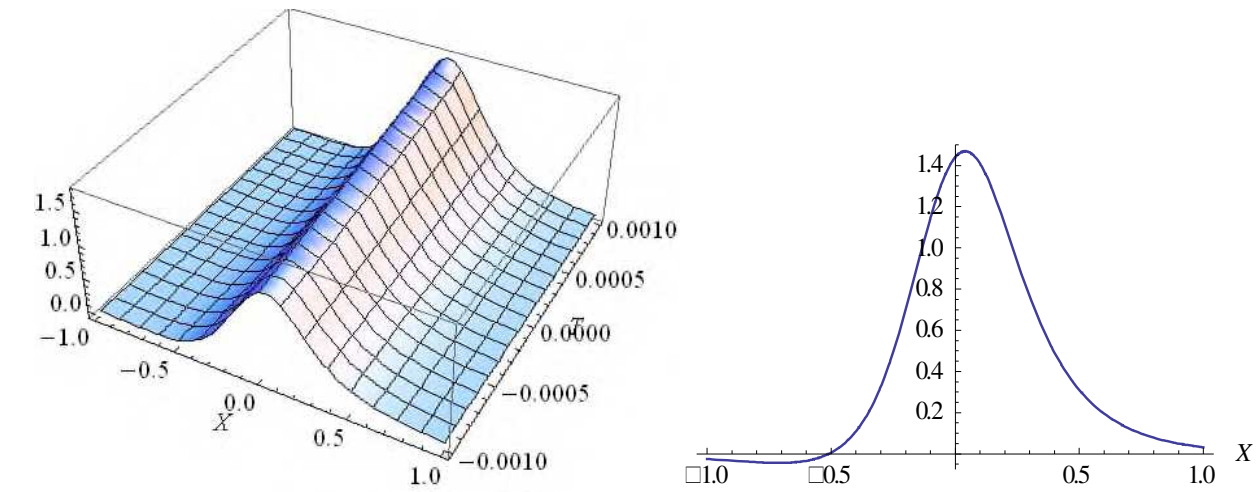

Fig. 7 : The bright cubic-quartic soliton for quadratic law the Re. part Eq.(50) in $2 \mathrm{D}$ and $3 \mathrm{D}$ with the values: $A_{1}=1.2-1.2 i, B=-2.3-0.3 i, \Omega=-7.9-201.7 i, a_{1}=-4, b_{1}=c_{1}=k_{1}=d_{1}=w_{1}=1, R_{1}=2$

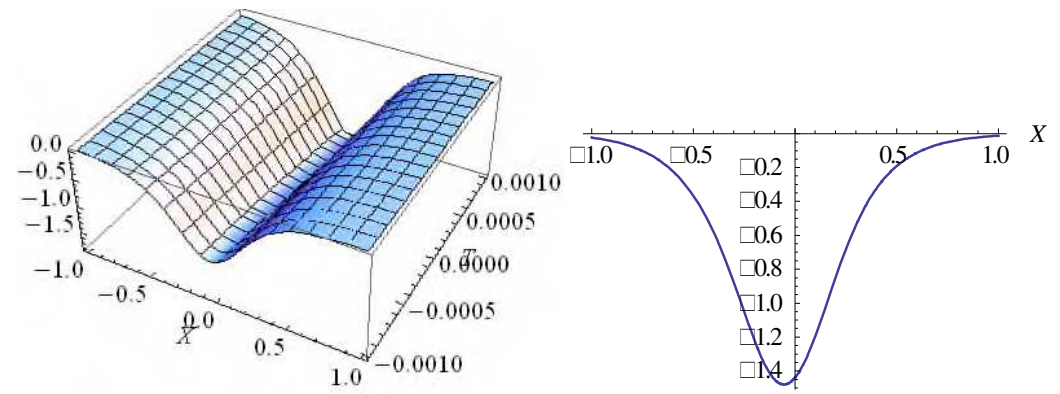

Fig. 8 : The bright cubic-quartic soliton for quadratic law the Im. part Eq.(51) in 2D and 3D with the values: $A_{1}=1.2-1.2 i, B=-2.3-0.3 i, \Omega=-7.9-201.7 i, a_{1}=-4, b_{1}=c_{1}=k_{1}=d_{1}=w_{1}=1, R_{1}=2$

\subsection{The dark cubic-quartic solitons}

Via inserting the relations (15-20) into the real and imaginary parts equations (41), (42) respectively we get,

$$
\begin{aligned}
& b_{1} A_{2} B^{3} R_{2}\left(R_{2}+1\right)\left(R_{2}+2\right)\left(R_{2}+3\right) \tanh ^{R_{2}+4}\left(t_{2}\right)+b_{1} A_{2} B^{3} R_{2}\left(R_{2}-1\right)\left(R_{2}-2\right)\left(R_{2}-3\right) \tanh ^{R_{2}-4} t_{2} \\
& -\left[b_{1} A_{2} B^{3} R_{2}\left(R_{2}-1\right)\left(3 R_{2}^{2}+8 R_{2}+8\right)+3 k\left(a_{1}+k\right) A_{2} R_{2}\left(R_{2}-1\right) B^{2}\right] \tanh ^{R_{2}-2} t_{2} \\
& +\left[2 b_{1} A_{2} B^{3} R_{2}\left(R_{2}+1\right)^{2}\left(R_{2}-2\right)-3 k\left(a_{1}+k\right) A_{2} R_{2}\left(R_{2}+1\right) B^{2}\right] \tanh ^{R_{2}+2} t_{2}+ \\
& {\left[\left(\Omega+k^{3}+k^{4}\right) A_{2}+6 k\left(a_{1}+k\right) A_{2} R_{2}^{2} B^{2}-b_{1} A_{2} B^{3} R_{2}\left(R_{2}^{4}+4 R_{2}^{3}+17 R_{2}^{2}+2 R_{2}+4\right)\right] \tanh ^{R_{2}} t_{2}} \\
& +2 c_{1} A_{2}^{2} \tanh ^{2 R_{2}} t_{2}+\left(e_{1}+d_{1}\right) A_{2}^{3} \tanh ^{3 R_{2}} t_{2}=0
\end{aligned}
$$




$$
\begin{aligned}
& {\left[A_{2} B R_{2} w_{2}+b_{1} k^{3} B A_{2} R_{2}\right] \tanh ^{R_{2}+1} t_{2}-\left[A_{2} B R_{2} w_{2}+b_{1} k^{3} B A_{2} R_{2}\right] \tanh ^{R_{2}-1} t_{2}} \\
& +\left(a_{1}+4 b_{1} k\right) A_{2} R_{2}\left(R_{2}+1\right) B^{2} \tanh ^{R_{2}+2} t_{2}+\left(a_{1}+4 b_{1} k\right) A_{2} R_{2}\left(R_{2}-1\right) B^{2} \tanh ^{R_{2}-2} t_{2} \\
& -\left(a_{1}+4 b_{1} k\right) 2 A_{2} R_{2}^{2} B^{2} \tanh ^{R_{2}} t_{2}=0
\end{aligned}
$$

When the equivalence between the higher orders of $\tanh ^{i} t_{2}$ is implemented at Eq. (52) and Eq.(53) the following relations, $\Omega+k^{4}+k^{3}+24 k\left(a_{1}+k\right) B^{2}-248 b_{1} B^{3}=0, a_{1}+k=-12 b_{1} B, R_{2}=2$,

$A_{2}^{2}=\frac{-120 b_{1} B^{3}}{e_{1}+d_{1}}, A_{2}=\frac{9\left(a_{1}+k\right) B^{2}}{c_{1}}, a_{1}=-4 k b_{1}$ and $w_{2}=-b_{1} k^{3}$ will be extracted, from which we get these values $A_{2}=1.4, b_{1}=-0.125, w_{2}=0.125, B=0.5, \Omega=-8.6$ after choosing $k_{1}=c_{1}=d_{1}=e_{1}=1$. Hence the solution in the framework of the proposed method and these achieved values of constants is:

$$
\begin{aligned}
& u(x, t)=A_{2} e^{i R_{2}(x, t)} \times \tanh ^{R_{2}} t_{2} \\
& u(x, t)=1.4 e^{i(x+8.6 t)} \times \tanh ^{2}[0.5(x-0.125 t)] \\
& \operatorname{Re} u(x, t)=1.4 \tanh ^{2}[(0.5 x-0.1 t)] \times \cos (x+8.6 t) \\
& \operatorname{Im} u(x, t)=1.4 \tanh ^{2}[(0.5 x-0.1 t)] \times \sin (x+8.6 t)
\end{aligned}
$$

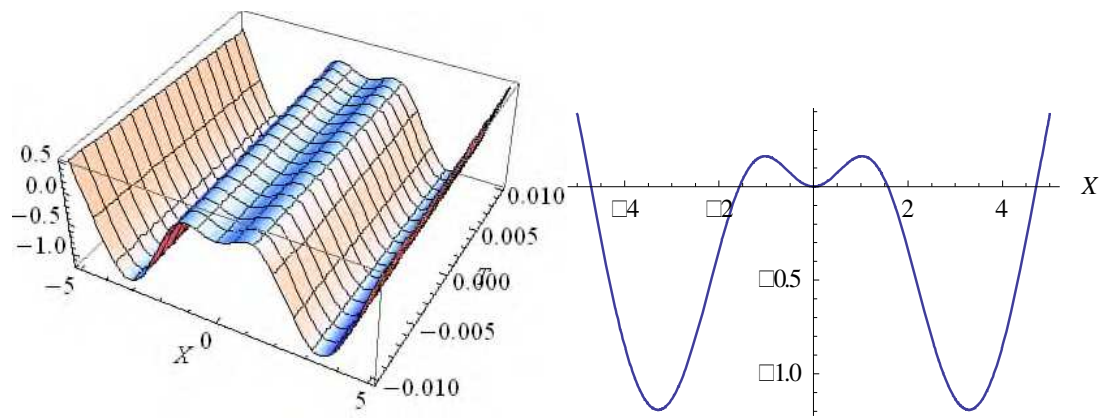

Fig. 9 : The dark cubic-quartic soliton for quadratic law the Re. part Eq.(55) in 2D and 3D with values

$$
A_{2}=1.4, a_{1}=0.5, b_{1}=-0.125, B=0.5, k_{1}=c_{1}=d_{1}=1, \Omega=-8.6, w_{2}=0.125, R_{2}=2
$$
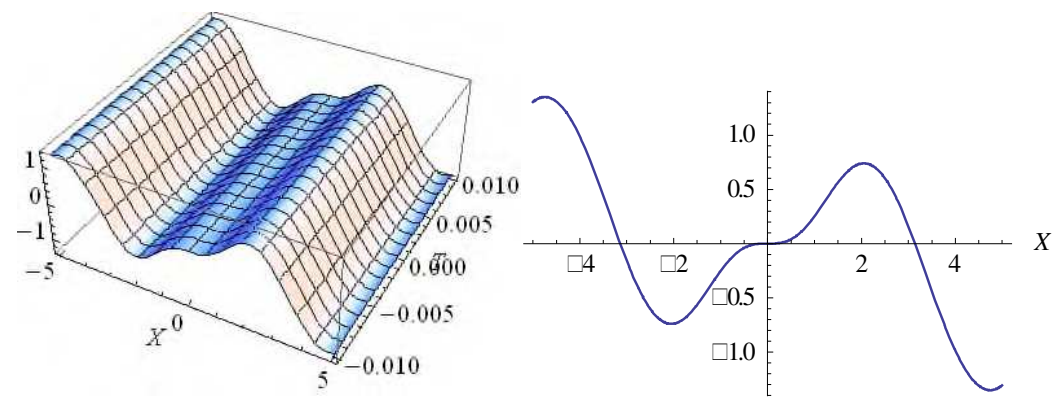

Fig. 10 : The dark cubic-quartic soliton for quadratic law the Im. part Eq.(56) in 2D and 3D with values: $A_{2}=1.4, a_{1}=0.5, b_{1}=-0.125, B=0.5, k_{1}=c_{1}=d_{1}=1, \Omega=-8.6, w_{2}=0.125, R_{2}=2$

\section{Application of the SWAM to the cubic-quartic for the Parabolic-Law NLSE in polarization-preserving fibers}

\subsection{The bright cubic-quartic solitons}

The cubic-quartic the NLSE in polarization-preserving fibers with parabolic law can be proposed in the form, 


$$
i Q_{t}+i a Q_{x x x}+b Q_{x x x x}=\left(c\left|Q^{2}\right|+d\left|Q^{4}\right|\right) Q
$$

The cubic-quartic NLSE in birefringent fiber for parabolic-law is,

$$
\begin{aligned}
& i u_{t}+i a_{1} u_{x x x}+b_{1} u_{x x x x}+c_{1}|u|^{2} u+d_{1}|v|^{2} u+\left(e_{1}\left|u^{4}\right|+g_{1}\left|u^{2}\right|\left|v^{2}\right|+h_{1}\left|v^{4}\right|\right) u=0 \\
& i v_{t}+i a_{1} v_{x x x}+b_{1} v_{x x x x}+c_{1}|v|^{2} v+d_{1}|u|^{2} v+\left(e_{1}\left|v^{4}\right|+g_{1}\left|v^{2}\right|\left|u^{2}\right|+h_{1}\left|u^{4}\right|\right) v=0
\end{aligned}
$$

Via inserting the relations (3-8) into any one of the two equations (58) or (59) mentioned above we get,

$$
\begin{aligned}
& i\left(\psi_{t}-i \Omega \psi\right) e^{i R_{1}}+i a_{1}\left(\psi_{x x x}+3 i k \psi_{x x}-i k^{3} \psi\right) e^{i R_{1}}+b_{1}\left(\psi_{x x x x}\right. \\
& \left.+4 i k \psi_{x x x}-3 k^{2} \psi_{x x}+k^{4} \psi-i k^{3} \psi\right) e^{i R_{1}}+\left(c_{1}+d_{1}\right) \psi^{3} e^{i R_{1}}+\left(e_{1}+g_{1}+h_{1}\right) \psi^{5} e^{i R_{1}}=0
\end{aligned}
$$

From which the following real and imaginary parts are emerges,

$$
\begin{aligned}
& \operatorname{Re} \Rightarrow b_{1} \psi_{x x x x}-3 k\left(a_{1}+k\right) \psi_{x x}+\left(\Omega+k^{3}+k^{4}\right) \psi+\left(c_{1}+d_{1}\right) \psi^{3}+\left(e_{1}+g_{1}+h_{1}\right) \psi^{5}=0 \\
& \operatorname{Im} \Rightarrow \psi_{t}+\left(a_{1}+4 b_{1} k\right) \psi_{x x x}-b_{1} k^{3} \psi_{x}=0
\end{aligned}
$$

Via inserting the relations (9-14) at the real and imaginary parts equations (61), (62) we get,

$$
\begin{aligned}
& {\left[b_{1} A_{1} B^{3} R_{1}\left(R_{1}+1\right)\left(R_{1}+2\right)-3 k\left(a_{1}+k\right) A_{1} B^{2} R_{1}\left(1+R_{1}\right)\right] \operatorname{sech}^{R_{1}+2} t_{1}} \\
& +\left[3 k\left(a_{1}+k\right) A_{1} B^{2} R_{1}^{2}+A_{1}\left(\Omega+k^{3}+k^{4}\right)-A_{1} B^{3} R_{1}^{4}\right] \operatorname{sech}^{R_{1}} t_{1} \\
& -A_{1} B^{3} R_{1}\left(R_{1}+1\right)^{2}\left(R_{1}+2\right) \operatorname{sech}^{R_{1}+4} t_{1}+\left(c_{1}+d_{1}\right) A_{1}^{3} \operatorname{sech}^{3 R_{1}} t_{1}+\left(e_{1}+g_{1}+h_{1}\right) A_{1}^{5} \operatorname{sech}^{5 R_{1}} t_{1}=0, \\
& \left(a_{1}+4 k b_{1}\right) A_{1} B^{3} R_{1}\left(R_{1}+1\right)\left(R_{1}+2\right) \operatorname{sech}^{R_{1}+2} t_{1} \tanh t_{1}-\left(a_{1}+4 k b_{1}\right) A_{1} B^{3} R_{1}^{3} \operatorname{sech}^{R_{1}} t_{1} \tanh t_{1}- \\
& b_{1} A R_{1} k^{3} B \operatorname{sech}^{R_{1}} t_{1} \tanh t_{1}-A_{1} B w_{1} R_{1} \operatorname{sech}^{R_{1}} t_{1} \tanh t_{1}=0,
\end{aligned}
$$

When the equivalence between the higher orders of $\operatorname{sech}^{i} t_{1}$ is implemented for Eq. (63) we extracting system of equations from which 6-differents results will emerged, for simplicity we will take only two of them which are,

$$
\begin{aligned}
& \text { (1) } A_{1}=0.24, B=1.3, \Omega=-41.6, R_{1}=1, w_{1}=1 \\
& \text { (2) } A_{1}=1.3+0.002 i, B=-(2.9+0.3 i), \Omega=-102-24.7 i, R_{1}=1, w_{1}=1
\end{aligned}
$$

The solution in the framework of the first result is,

$$
\begin{aligned}
& u=0.24 \operatorname{sech}[1.3(x-t)] e^{i(x+41.6 t)} \\
& \operatorname{Re} u=0.24 \operatorname{sech}[1.3 x-1.3 t] \times \cos (x+41.6 t) \\
& \operatorname{Im} u=0.24 \operatorname{sech}[1.3 x-1.3 t] \times \sin (x+41.6 t)
\end{aligned}
$$
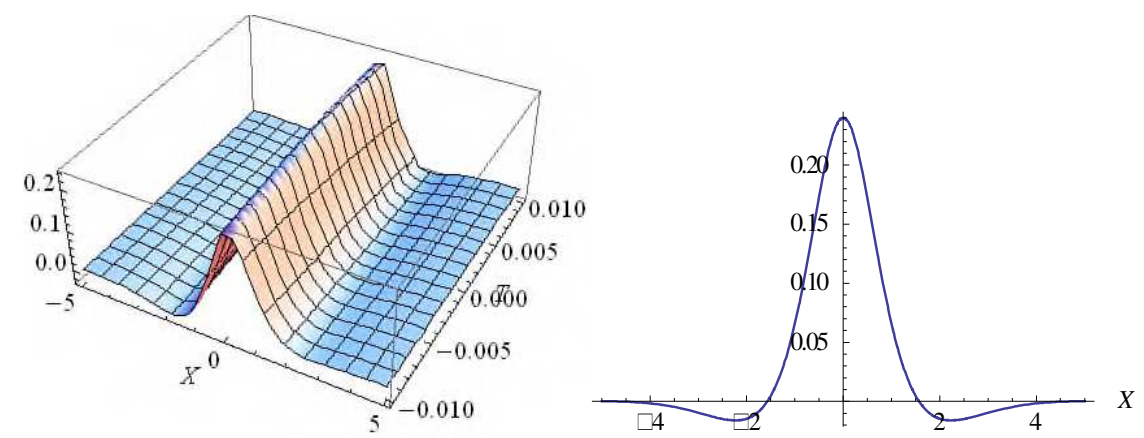

Fig. 11 : The bright cubic-quartic soliton for parabolic law the Re. part Eq.(67) in 2D and 3D with $A_{1}=0.24, B=1.2, \Omega=-41.6, a_{1}=-4, b_{1}=c_{1}=e_{1}=g_{1}=h_{1}=k_{1}=d_{1}=w_{1}=1, R_{1}=1$ 


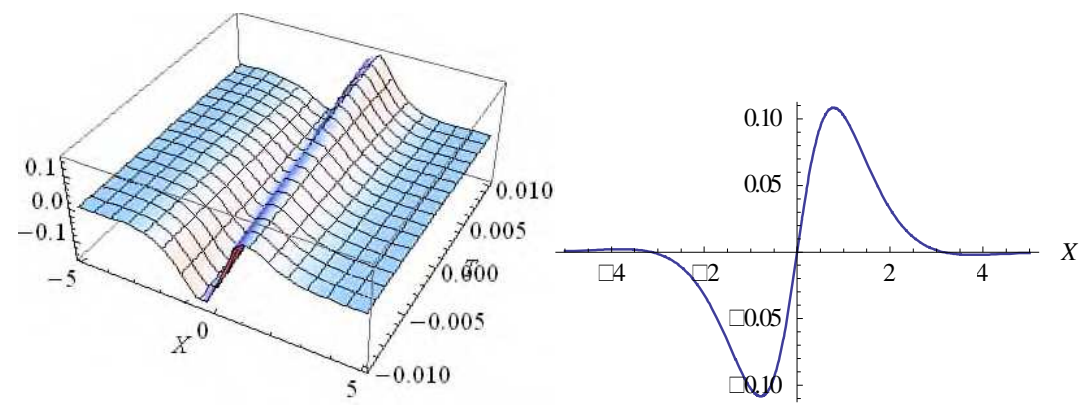

Fig. 12 : The bright cubic-quartic soliton for parabolic law the Im. part Eq.(68) in 2D and 3D with $A_{1}=0.24, B=1.2, \Omega=-41.6, a_{1}=-4, b_{1}=c_{1}=e_{1}=g_{1}=h_{1}=k_{1}=d_{1}=w_{1}=1, R_{1}=1$

While the solution in the framework of the second result is,

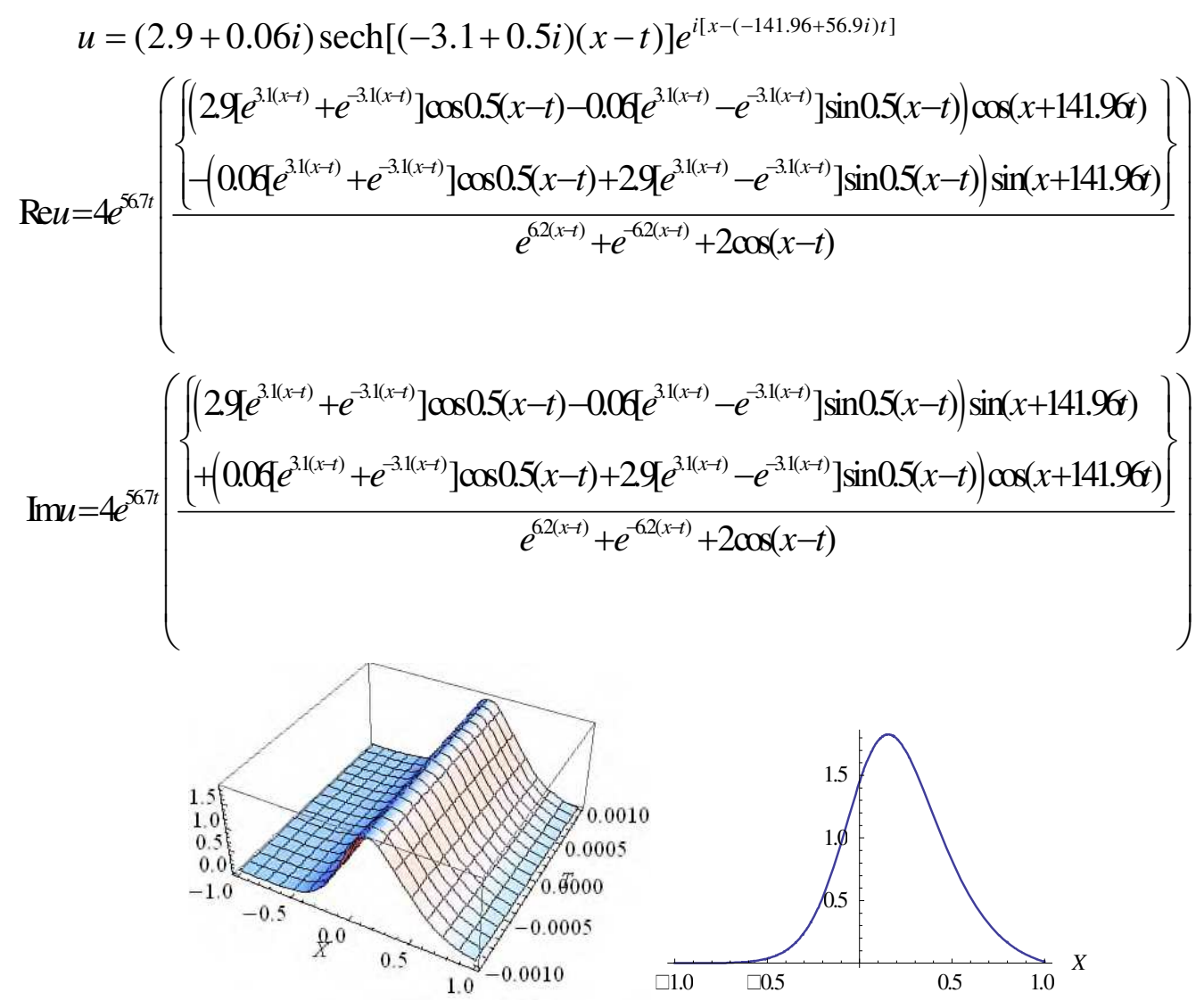

Fig. 13 : The bright cubic-quartic soliton for Parabolic law the Re. part Eq.(70) in 2D and 3D with $A_{1}=1.3+0.002 i, B=-(2.9+0.3 i), \Omega=-102-24.7 i, a_{1}=-4, b_{1}=c_{1}=k_{1}=d_{1}=w_{1}=1, R_{1}=1$
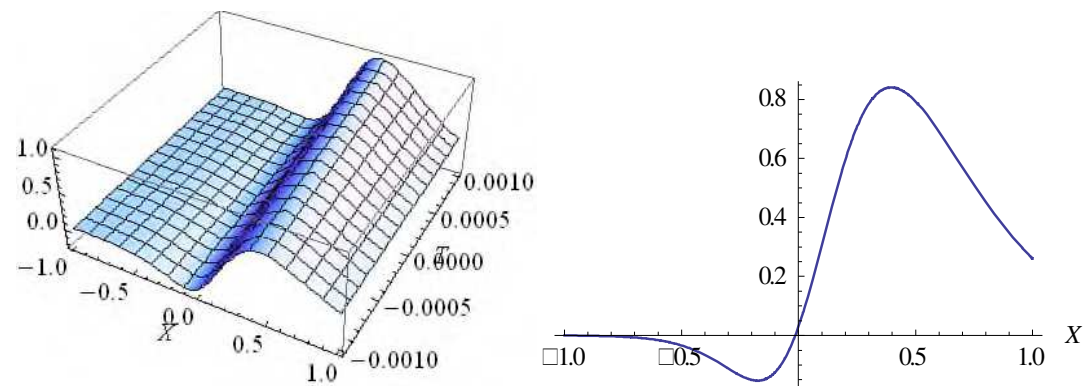

Fig. 14 : The bright cubic-quartic soliton for parabolic law the Im. part Eq.(71) in 2D and 3D with $A_{1}=1.3+0.002 i, B=-(2.9+0.3 i), \Omega=-102-24.7 i, a_{1}=-4, b_{1}=c_{1}=k_{1}=d_{1}=w_{1}=1, R_{1}=1$

\subsection{The dark cubic soliton for the parabolic-law nonlinearity}

Via inserting the relations (15-20) into the real and imaginary parts equations (61), (62) 
respectively we get,

$$
\begin{aligned}
& b_{1} A_{2} B^{3} R_{2}\left(R_{2}+1\right)\left(R_{2}+2\right)\left(R_{2}+3\right) \tanh ^{R_{2}+4}\left(t_{2}\right)+b_{1} A_{2} B^{3} R_{2}\left(R_{2}-1\right)\left(R_{2}-2\right)\left(R_{2}-3\right) \tanh ^{R_{2}-4} t_{2} \\
& -\left[b_{1} A_{2} B^{3} R_{2}\left(R_{2}-1\right)\left(3 R_{2}^{2}+8 R_{2}+8\right)+3 k\left(a_{1}+k\right) A_{2} R_{2}\left(R_{2}-1\right) B^{2}\right] \tanh ^{R_{2}-2} t_{2} \\
& +\left[2 b_{1} A_{2} B^{3} R_{2}\left(R_{2}+1\right)^{2}\left(R_{2}-2\right)-3 k\left(a_{1}+k\right) A_{2} R_{2}\left(R_{2}+1\right) B^{2}\right] \tanh ^{R_{2}+2} t_{2}+ \\
& {\left[\left(\Omega+k^{3}+k^{4}\right) A_{2}+6 k\left(a_{1}+k\right) A_{2} R_{2}^{2} B^{2}-b_{1} A_{2} B^{3} R_{2}\left(R_{2}^{4}+4 R_{2}^{3}+17 R_{2}^{2}+2 R_{2}+4\right)\right] \tanh ^{R_{2}} t_{2}} \\
& +\left(c_{1}+d_{1}\right) A_{2}^{3} \tanh ^{3 R_{2}} t_{2}+\left(e_{1}+g_{1}+h_{1}\right) A_{2}^{5} \tanh ^{5 R_{2}} t_{2}=0 \\
& {\left[A_{2} B R_{2} w_{2}+b_{1} k^{3} B A_{2} R_{2}\right] \tanh ^{R_{2}+1} t_{2}-\left[A_{2} B R_{2} w_{2}+b_{1} k^{3} B A_{2} R_{2}\right] \tanh ^{R_{2}-1} t_{2}} \\
& +\left(a_{1}+4 b_{1} k\right) A_{2} R_{2}\left(R_{2}+1\right) B^{2} \tanh ^{R_{2}+2} t_{2}+\left(a_{1}+4 b_{1} k\right) A_{2} R_{2}\left(R_{2}-1\right) B^{2} \tanh ^{R_{2}-2} t_{2} \\
& -\left(a_{1}+4 b_{1} k\right) 2 A_{2} R_{2}^{2} B^{2} \tanh ^{R_{2}} t_{2}=0
\end{aligned}
$$

When the equivalence between the higher orders of $\tanh ^{i} t_{2}$ is implemented for Eq.(72) and via using Eq.(73) the following relations, $R_{2}=2, \Omega+k^{4}+k^{3}+6 k\left(a_{1}+k\right) B^{2}-28 b_{1} B^{3}=0$, $A_{2}^{4}=\frac{-24 b_{1} B^{3}}{e_{1}+g_{1}+h_{1}},\left(c_{1}+d_{1}\right) A_{2}^{2}-6\left(a_{1}+k\right) B^{2}-8 b_{1} B^{3}=0, a_{1}=-4 k b_{1}, w_{2}=-b_{1} k^{3}$, via solving these equations after choosing $c_{1}=d_{1}=e_{1}=g_{1}=h_{1}=1,6$-solutions will be extracted, for simplicity and similarity we will plot only two of them which are,

$$
\begin{aligned}
& \text { (1) } A_{2}=-0.4 i, B=-0.1, \Omega=-3.6, w_{2}=-1 \\
& \text { (2) } A_{2}=3.9-2.9 i, B=2.3+0.4 i, \Omega=401-206 i, w_{2}=-1
\end{aligned}
$$

The solution of the first result in the framework of the suggested method is,

$$
\begin{aligned}
& u(x, t)=A_{2} e^{i R_{2}(x, t)} \times \tanh ^{R_{2}} t_{2} \\
& u(x, t)=-0.4 i e^{i(x+3.6 t)} \times \tanh [-0.1(x+t)] \\
& \operatorname{Re} u(x, t)=0.4 \times \sin (x+3.6 t) \times \tanh [-0.1(x+t)] \\
& \operatorname{Im} u(x, t)=-0.4 \times \cos (x+3.6 t) \times \tanh [-0.1(x+t)]
\end{aligned}
$$
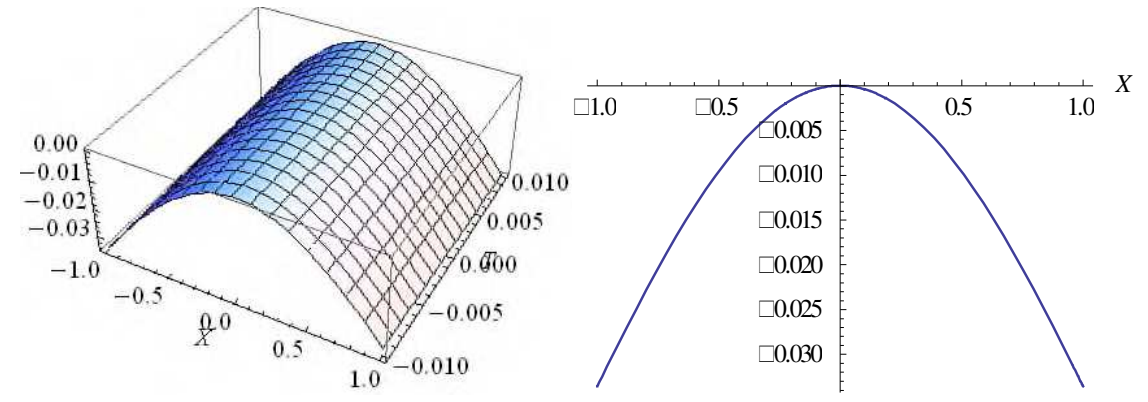

Fig. 15 : The dark cubic-quartic soliton for parabolic law the Re. part Eq.(76) in 2D and 3D with values: $A_{2}=-0.4 i, B=-0.1, \Omega=-3.6, w_{2}=-1, a_{1}=-4, b_{1}=k_{1}=c_{1}=e_{1}=g_{1}=h_{1}=1, R_{2}=1$ 

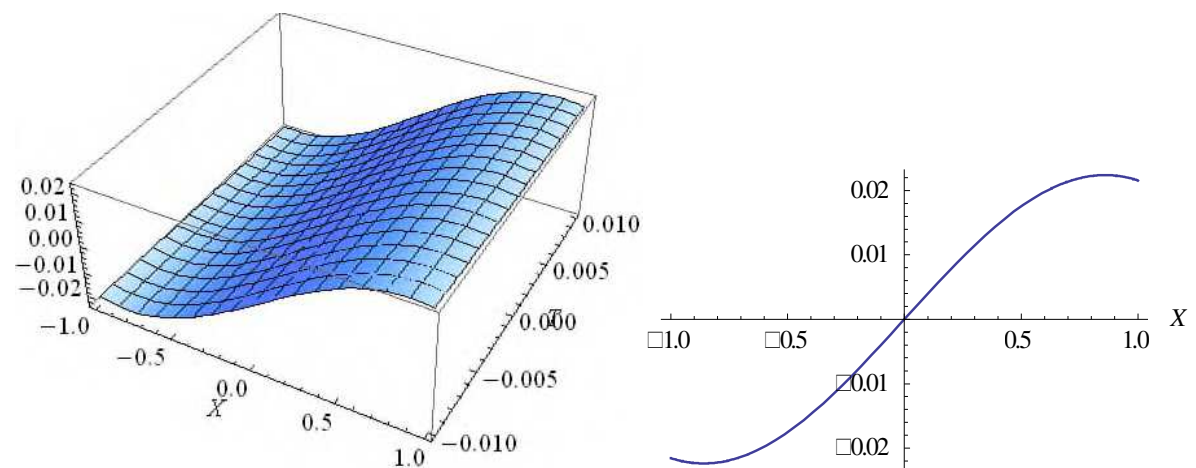

Fig. 16 : The dark cubic-quartic soliton for parabolic law the Im. part Eq.(77) in 2D and 3D with values: $A_{2}=-0.4 i, B=-0.1, \Omega=-3.6, w_{2}=-1, a_{1}=-4, b_{1}=k_{1}=c_{1}=e_{1}=g_{1}=h_{1}=1, R_{2}=1$

The solution of the first result in the framework of the suggested method is,

$$
\begin{aligned}
& u(x, t)=A_{2} e^{i R_{2}(x, t)} \times \tanh ^{R_{2}} t_{2} \\
& u(x, t)=(3.9-2.9 i) \times e^{i[x-(401-206 i) t]} \times \tanh [(2.3+0.4 i)(x+t)] \\
& \operatorname{Re} u(x, t)=e^{-206 t} \frac{\left\{\begin{array}{l}
\left(e^{9.2(x+t)}-1\right)(3.9 \cos (x-401 t)+2.9 \sin (x-401 t)) \\
-2 e^{4.6(x+t)}(3.9 \sin (x-401 t)-2.9 \cos (x-401 t)) \sin 0.8(x+t)
\end{array}\right\}}{1+e^{9.2(x+t)}+2 e^{4.6(x+t)} \cos 0.8(x+t)} \\
& \operatorname{Im} u(x, t)=e^{-206 t} \frac{\left\{\begin{array}{l}
2 e^{4.6(x+t)} \sin 0.8(x+t)(3.9 \cos (x-401 t)+2.9 \sin (x-401 t)) \\
+\left(e^{9.2(x+t)}-1\right)(3.9 \sin (x-401 t)-2.9 \cos (x-401 t))
\end{array}\right\}}{1+e^{9.2(x+t)}+2 e^{4.6(x+t)} \cos 0.8(x+t)} \\
& \text { (1.0) }
\end{aligned}
$$

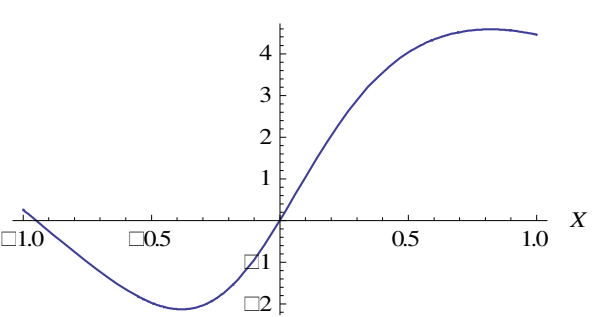

Fig. 17 : The dark cubic-quartic soliton for parabolic law the Re. part Eq.(79) in 2D and 3D with values: $A_{2}=3.9-29 i, B=23+0.4 i, \Omega=401-206 i, w_{2}=-1, a_{1}=-4, b_{1}=k_{1}=c_{1}=e_{1}=g_{1}=h_{1}=1, R_{2}=1$
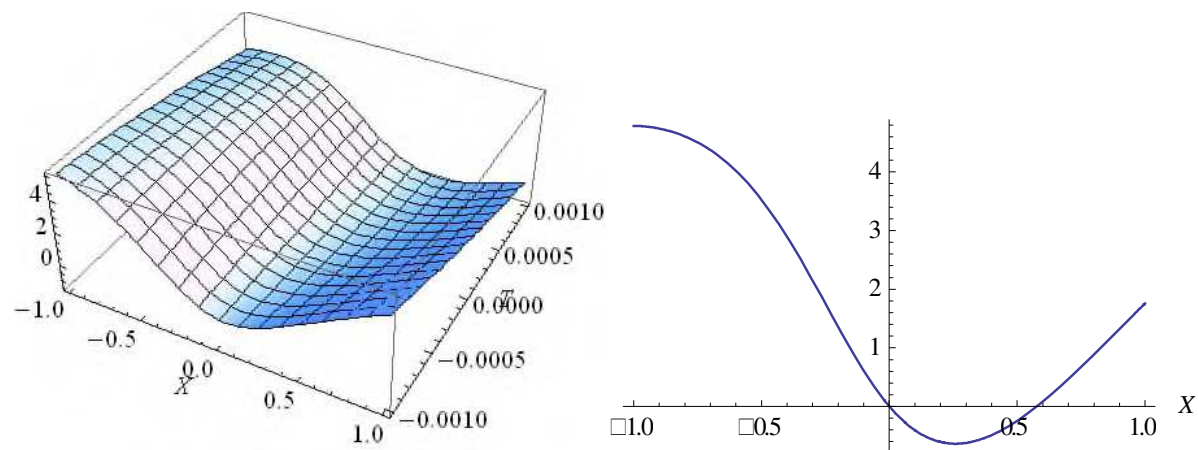

Fig. 18 : The dark cubic-quartic soliton for Parabolic law the Im. part Eq.(80) in 2D and 3D with values: $A_{2}=3.9-29 i, B=23+0.4 i, \Omega=401-206 i, w_{2}=-1, a_{1}=-4, b_{1}=k_{1}=c_{1}=e_{1}=g_{1}=h_{1}=1, R_{2}=1$ 


\section{Application of the SWAM to the cubic-quartic for the Non-Local-Law NLSE in polarization-preserving fibers \\ 6.1 The bright cubic-quartic solitons}

The cubic-quartic the NLSE in polarization-preserving fibers with non-local law can be proposed in the form,

$$
i Q_{t}+i a Q_{x x x}+b Q_{x x x x}+\left(c\left|Q^{2}\right|\right)_{x x} Q=0
$$

The cubic-quartic NLSE in birefringent fiber non-local Law is,

$$
\begin{aligned}
& i u_{t}+i a_{1} u_{x x x}+b_{1} u_{x x x x}+\left(c_{1}\left(|u|^{2}\right)_{x x}+d_{1}\left(|v|^{2}\right)_{x x}\right) u=0 \\
& i v_{t}+i a_{2} v_{x x x}+b_{1} v_{x x x x}+\left(c_{2}\left(|v|^{2}\right)_{x x}+d_{2}\left(|u|^{2}\right)_{x x}\right) v=0
\end{aligned}
$$

Via inserting the relations (3-8) into any one of the two equations (82) or (83) mentioned above we get,

$$
\begin{aligned}
& i\left(\psi_{t}-i \Omega \psi\right) e^{i R_{1}}+i a_{1}\left(\psi_{x x x}+3 i k \psi_{x x}-i k^{3} \psi\right) e^{i R_{1}}+b_{1}\left(\psi_{x x x}\right. \\
& \left.+4 i k \psi_{x x x}-3 k^{2} \psi_{x x}+k^{4} \psi-i k^{3} \psi\right) e^{i R_{1}}+\left(2 c_{1}+2 d_{1}\right) \psi \psi_{x}^{2} e^{i R_{1}} \\
& +\left(2 c_{1}+2 d_{1}\right) \psi \psi_{x x} e^{i R_{1}}=0
\end{aligned}
$$

From which the following real and imaginary parts are emerges,

$$
\begin{aligned}
& \operatorname{Re} \Rightarrow b_{1} \psi_{x x x}-3 k\left(a_{1}+k\right) \psi_{x x}+\left(\Omega+k^{3}+k^{4}\right) \psi+\left(2 c_{1}+2 d_{1}\right) \psi \psi_{x}^{2}+\left(2 c_{1}+2 d_{1}\right) \psi \psi_{x x}=0 \\
& \operatorname{Im} \Rightarrow \psi_{t}+\left(a_{1}+4 b_{1} k\right) \psi_{x x x}-b_{1} k^{3} \psi_{x}=0
\end{aligned}
$$

Via inserting the relations (9-14) into the real and imaginary parts equations (85), (86) we get,

$$
\begin{aligned}
& {\left[b_{1} A_{1} B^{3} R_{1}\left(R_{1}+1\right)\left(R_{1}+2\right)-3 k\left(a_{1}+k\right) A_{1} B^{2} R_{1}\left(1+R_{1}\right)\right] \operatorname{sech}^{R_{1}+2} t_{1}} \\
& +\left[3 k\left(a_{1}+k\right) A_{1} B^{2} R_{1}^{2}+A_{1}\left(\Omega+k^{3}+k^{4}\right)-A_{1} B^{3} R_{1}^{4}\right] \operatorname{sech}^{R_{1}} t_{1} \\
& -A_{1} B^{3} R_{1}\left(R_{1}+1\right)^{2}\left(R_{1}+2\right) \operatorname{sech}^{R_{1}+4} t_{1}+\left(2 c_{1}+2 d_{1}\right) A_{1}^{3} B^{2} R_{1}^{2}\left[\operatorname{sech}^{2 R_{1}} t_{1}-\operatorname{sech}^{2 R_{1}+2} t_{1}\right] \\
& +\left(2 c_{1}+2 d_{1}\right)\left[A_{1}^{2} B^{2} R_{1}\left(R_{1}+1\right) \operatorname{sech}^{2 R_{1}+2} t_{1}-A_{1}^{2} B^{2} R_{1}^{2} \operatorname{sech}^{2 R_{1}} t_{1}\right]=0 \\
& \left(a_{1}+4 k b_{1}\right) A_{1} B^{3} R_{1}\left(R_{1}+1\right)\left(R_{1}+2\right) \operatorname{sech}^{R_{1}+2} t_{1} \tanh t_{1}-\left(a_{1}+4 k b_{1}\right) A B^{3} R_{1}^{3} \operatorname{sech}^{R_{1}} t_{1} \tanh t_{1}- \\
& b_{1} A_{1} R_{1} k^{3} B \operatorname{sech}^{R_{1}} t_{1} \tanh t_{1}-A_{1} B w_{1} R_{1} \operatorname{sech}^{R_{1}} t_{1} \tanh t_{1}=0,
\end{aligned}
$$

When the equivalence between the higher orders of $\operatorname{sech}^{i} t_{1}$ is implemented for Eq. (87) we extracting system of equations from which 6-differents results will emerged, for simplicity we will take only one of them which is,

$$
\begin{gathered}
A_{1}=2+0.8 i, B=-(0.1+0.5 i), \Omega=-8+5 i, R_{1}=2, a_{1}=-4, b_{1}=c_{1}=k_{1}=d_{1}=w_{1}=1 \\
u=e^{5 t}(2+0.8 i) \operatorname{sech}^{2}[-(0.1+0.5 i)(x-t)] e^{i(x+8 t)} \\
\operatorname{Re} u=4 e^{5 t}\left\{\begin{array}{l}
\left\{\begin{array}{l}
(2 \cos (x+8 t)-0.8 \sin (x+8 t))\left(2+e^{02(x-t)}+e^{-02(x-t)} \cos (x-t)+2 \sin ^{2}(0.5 x-0.5 t) \cos ^{2}(0.5 x-0.5 t)\right) \\
+\sin (x-t)[-0.8 \cos (x+8 t)+2 \sin (x+8 t)]\left(e^{02(x-t)}-e^{-02(x-t)}\right)
\end{array}\right\} \\
\left.\left\{\begin{array}{l}
4+\cos (x-t)\left[2+4 e^{-02(x-t)}+2 \sin ^{2}(0.5 x-0.5 t) \cos ^{2}(0.5 x-0.5 t)\right]+\sin ^{2}(0.5 x-0.5 t)\left(e^{04(x-t)}-2\right) \\
+4 \sin ^{2}(0.5 x-0.5 t) \cos ^{2}(0.5 x-0.5 t)\left(1+e^{02(x-t)}\right)+e^{-0.4(x-t)}+5 e^{02(x-t)} 4 \sin ^{4}(0.5 x-0.5 t) \cos ^{4}(0.5 x-0.5 t)
\end{array}\right\}\right)
\end{array}\right.
\end{gathered}
$$




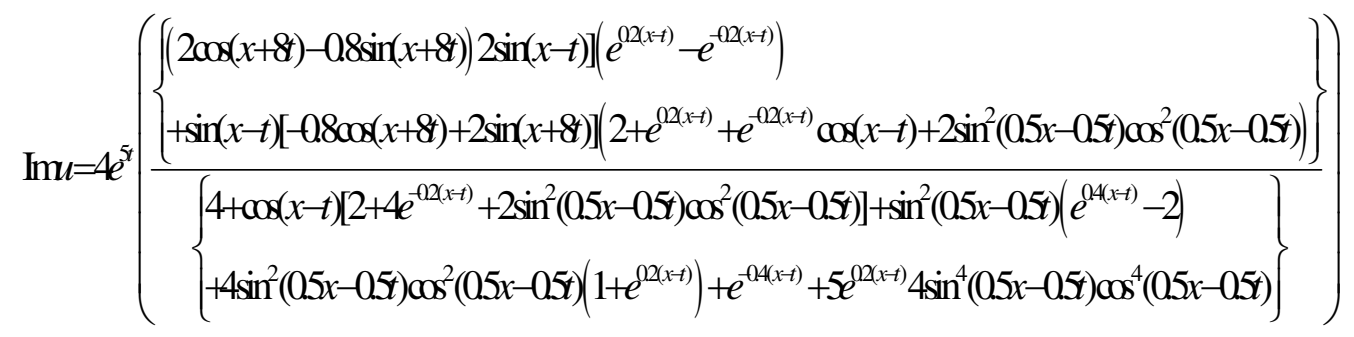

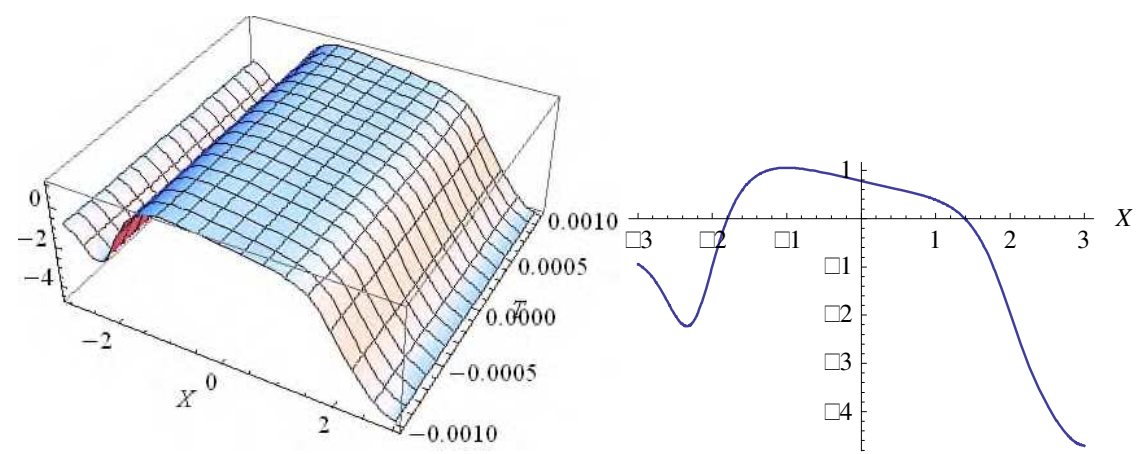

Fig. 19 : The bright cubic-quartic soliton for non-local law the Re. part Eq.(91) in 2D and 3D with values $A_{1}=2+0.8 i, B=-(0.1+0.5 i), \Omega=-8+5 i, R_{1}=2, a_{1}=-4, b_{1}=c_{1}=k_{1}=d_{1}=w_{1}=1$

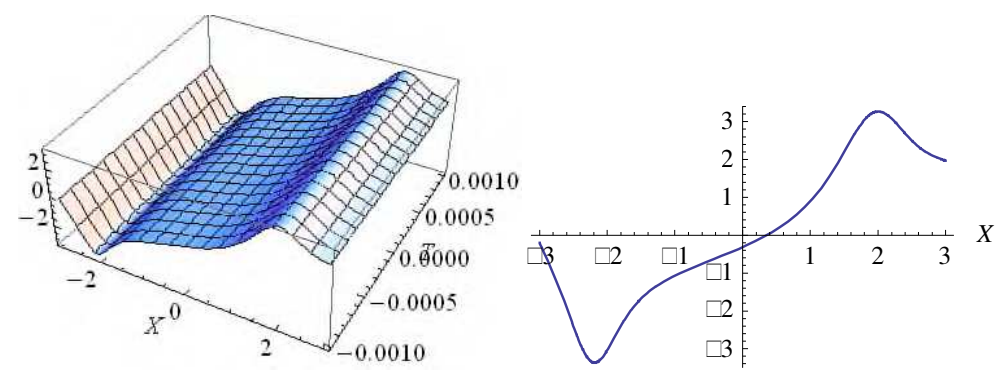

Fig. 20 : The bright cubic-quartic soliton for non-local law the Im. part Eq.(92) in 2D and 3D with values $A_{1}=2+0.8 i, B=-(0.1+0.5 i), \Omega=-8+5 i, R_{1}=2, a_{1}=-4, b_{1}=c_{1}=k_{1}=d_{1}=w_{1}=1$

\subsection{The dark cubic soliton for the non-local-law nonlinearity}

Via inserting the relations (15-20) into the real and imaginary parts equations (85), (86) respectively we get,

$$
\begin{aligned}
& b_{1} A_{2} B^{3} R_{2}\left(R_{2}+1\right)\left(R_{2}+2\right)\left(R_{2}+3\right) \tanh ^{R_{2}+4} t_{2}+b_{1} A_{2} B^{3} R_{2}\left(R_{2}-1\right)\left(R_{2}-2\right)\left(R_{2}-3\right) \tanh ^{R_{2}-4} t_{2} \\
& +\left[2 b_{1} A_{2} B^{3} R_{2}\left(R_{2}+1\right)^{2}\left(R_{2}-2\right)+A_{2} R_{2}\left(R_{2}+1\right) B^{2}\right] \tanh ^{R_{2}+2} t_{2} \\
& -\left[b_{1} A_{2} B^{3} R_{2}\left(R_{2}-1\right)\left(3 R_{2}^{2}+8 R_{2}+8\right)+3 k\left(a_{1}+k\right) A_{2} R_{2}\left(R_{2}-1\right) B^{2}\right] \tanh ^{R_{2}-2} t_{2} \\
& +\left[\left(\Omega+k^{3}+k^{4}\right) A_{2}+6 k\left(a_{1}+k\right) A_{2} R_{2}^{2} B^{2}-b_{1} A_{2} B^{3} R_{2}\left(R_{2}^{4}+4 R_{2}^{3}+17 R_{2}^{2}+2 R_{2}+4\right)\right] \tanh ^{R_{2}} t_{2} \\
& +\left(2 c_{1}+2 d_{1}\right) A_{2}^{3} B^{3} R_{2}^{2} \tanh ^{3 R_{2}-2} t_{2}-\left(2 c_{1}+2 d_{1}\right) A_{2}^{3} B^{3} R_{2}^{2} \tanh ^{3 R_{2}+2} t_{2}-2\left(2 c_{1}+2 d_{1}\right) A_{2}^{3} B^{3} R_{2}^{2} \tanh ^{3 R_{2}} t_{2} \\
& +\left(2 c_{1}+2 d_{1}\right) A_{2}^{2} R_{2}\left(R_{2}-1\right) B^{2} \tanh ^{2 R_{2}-2} t_{2}-2\left(2 c_{1}+2 d_{1}\right) B^{2} A_{2}^{2} R_{2}^{2} \tanh ^{2 R_{2}} t_{2} \\
& +\left(2 c_{1}+2 d_{1}\right) A_{2}^{2} R_{2}\left(R_{2}+1\right) \tanh ^{2 R_{2}+2} t_{2}=0 \\
& {\left[A_{2} B R_{2} w_{2}+b_{1} k^{3} B A_{2} R_{2}\right] \tanh ^{R_{2}+1} t_{2}-\left[A_{2} B R_{2} w_{2}+b_{1} k^{3} B A_{2} R_{2}\right] \tanh ^{R_{2}-1} t_{2}} \\
& +\left(a_{1}+4 b_{1} k\right) A_{2} R_{2}\left(R_{2}+1\right) B^{2} \tanh ^{R_{2}+2} t_{2}+\left(a_{1}+4 b_{1} k\right) A_{2} R_{2}\left(R_{2}-1\right) B^{2} \tanh ^{R_{2}-2} t_{2} \\
& -\left(a_{1}+4 b_{1} k\right) 2 A_{2} R_{2}^{2} B^{2} \tanh ^{R_{2}} t_{2}=0
\end{aligned}
$$


When the equivalence between the higher orders of $\tanh ^{i} t_{2}$ is implemented for Eq.(93) and by using Eq.(94) the following relations, $\Omega+k^{4}+k^{3}+6 k\left(a_{1}+k\right) B^{2}-28 b_{1} B^{3}+2\left(c_{1}+d_{1}\right) A_{2}^{2} B^{3}=0, R_{2}=1$,

$$
A_{2}^{2}=\frac{12 b_{1}}{c_{1}+d_{1}},\left(c_{1}+d_{1}\right) A_{2}^{2}-6\left(a_{1}+k\right) B^{2}-8 b_{1} B^{3}=0, a_{1}=-4 k b_{1} \text { and } w_{2}=-b_{1} k^{3} \text { will } \quad \text { be } \quad \text { extracted }
$$

after choosing $b_{1}=c_{1}=d_{1}=k=1$, hence $B=0.04, A_{2}= \pm \sqrt{6}, \Omega=-2.02, w_{2}=-1$.

This result will generate two solutions according to the sign of $A_{2}$.

The solutions according values and in the framework of the proposed method these is,

$$
\begin{aligned}
& u(x, t)=A_{2} e^{i R_{2}(x, t)} \times \tanh ^{R_{2}} t_{2} \\
& u(x, t)= \pm \sqrt{6} e^{i(x+2.02) t} \times \tanh 0.04(x+t) \\
& \operatorname{Re} u(x, t)= \pm \sqrt{6} \cos (x+2.02 t) \times \tanh 0.04(x+t) \\
& \operatorname{Im} u(x, t)= \pm \sqrt{6} \sin (x+2.02 t) \times \tanh 0.04(x+t)
\end{aligned}
$$

For simplicity we will the positive result only and plot it
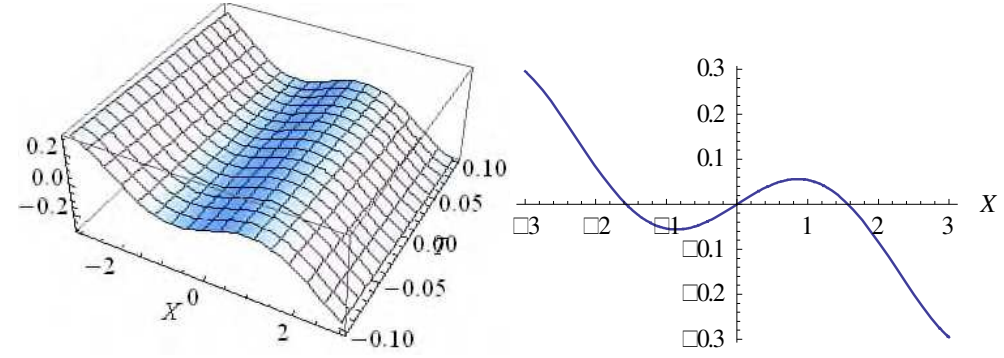

Fig. 21 : The dark cubic-quartic soliton for non-local law the Re. part Eq.(96) in 2D and 3D with values $A_{2}=2.5, B=0.04, \Omega=-2.02, R_{2}=1, a_{1}=-4, b_{1}=c_{1}=k_{1}=d_{1}=w_{1}=-1$

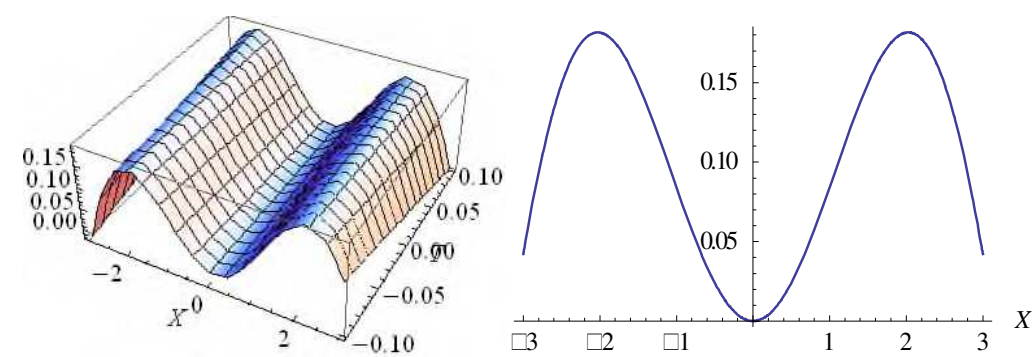

Fig. 22 : The dark cubic-quartic soliton for non-local law the Im. part Eq.(97) in 2D and 3D with values $A_{2}=2.5, B=0.04, \Omega=-2.02, R_{2}=1, a_{1}=-4, b_{1}=c_{1}=k_{1}=d_{1}=w_{1}=-1$

\section{Conclusions:}

Through this paper, new multiple important and impressive perceptions of the four forms of the nonlinear refractive index cubic-quartic through birefringent fibers "which weren't achieved before by any other authors who used different techniques" have been emerged via the SWAM. The four distinct forms of the cubic-quartic NLSE in polarization-preserving are: the cubic quartic of the kerr-law NLSE, the cubic quartic of the quadratic-law NLSE, the cubic quartic of the parabolic-law NLSE and the cubic quartic of the non-local-law NLSE. The suggested method has been applied perfectly to construct multiple visions for each one of the above mentioned cases individually by the same path at the same time and parallel. In the Figs. (1-4), we documented the solutions forms behavior which is achieved for the cubic 
quartic kerr-law NLSE, while the Figs. (5-10) illustrate the significant solutions behavior for the cubic quartic of the quadratic-law NLSE, moreover the Figs. (11-18) represent the behavior of the distinct solutions forms of the cubic quartic of the parabolic-law NLSE, finally the Figs.(19-22) demonstrate good description of the solutions of the cubic quartic of the non-local-law NLSE. Most of the achieved optical solitons for the four different proposed forms are new compared by [17-19], [28-30] who used various techniques to study these four forms. Consequently, through this work we can introduce future study not only for these four various forms but also for all related phenomenon's have been demonstrated.

\section{References:}

[1] Erasmus Bartholin, Experimenta crystalli islandici disdiaclastici quibus mira \& infolita refractio detegitur [Experiments on birefringent Icelandic crystal through which is detected a remarkable and unique refraction] (Copenhagen, Denmark: Daniel Paulli, 1669).

[2] Neves, N. M. (1998); The use of birefringence for predicting the stiffness of injection molded polycarbonate discs. Polymer Engineering \& Science. 38 (10): 1770-1777.

[3] Brad Amos. Birefringence for facetors I: what is birefringence? Archived December 14, 2013, at the Wayback Machine First published in Stone Chat, the Journal of the UK Facet Cutter's Guild. January-March. edition (2005)

[4] Nofal, T.A., (2016); Simple equation method for nonlinear partial differential equations and its applications, J. Egypt. Math. Soc. 24, 204-209.

[5] Kudryashov, N.A., (2020); Mathematical model of propagation pulse in optical fiber with power nonlinearities, Optik 212;164750.

[6] Kudryashov, N.A., (2020); Highly dispersive solitary wave solutions of perturbed nonlinear Schrödinger equations, Appl. Math. Comput. 371; 124972.

[7] Kudryashov, N.A., (2020); Highly dispersive optical solitons of the generalized nonlinear eighth-order Schrödinger equation, Optik 206;164335.

[8] Li, B., Zhao, J., Pan, A., Mirzazadeh, M.,Ekici, M.,Zhou, Q., Liu, W., (2019); Stable propagation of optical solitons in fiber lasers by using symbolic computation, Optik 178;142145.

[9] Liu, C., (2005); Exact solutions for the higher-order nonlinear Schrodinger equation in nonlinear optical fibres. Chaos, Solitons Fractals 23, 949-955.

[10] Xiao, L.L., Liang, W.M., (2009); The $\left(\mathrm{G}^{\prime} / \mathrm{G}\right)$-Expansion Method and travelling wave solutions for a higher-order nonlinear Schrödinger equation, Appl. Math. Comput. 208, 440445.

[11] El-Ganaini, S.I.A., (2013); The first integral method to the nonlinear Schrodinger equations in higher dimensions, Abst. Appl. Anal., 10, 349173.

[12] Mirzazadeh, M., Eslami, M., Biswas, A., (2014); Dispersive optical solitons by Kudryashov's method; Optik; 125, 23, 6874-6880

[13] Shehata, M.S.M., Rezazadeh, H., Jawad, A.J.M., Zahran, E.H.M., Bekir, A., (2021); Optical solitons to a perturbed Gerdjikov-Ivanov equation using two different techniques, Revista Mexicana de Física, 67; 050704 1-15

[14] Bekir, A., Zahran, E.H.M., (2021); New visions of the soliton solutions to the modified nonlinear Schrodinger equation, Optik, 232, 166539

[15] Blanco-Redondo, A., Sterke, C.M.D., Sipe, J.E., Krauss, T.F., Eggleton, B.J., Husko, C., (2016); Pure-quartic solitons, Nature Communications 7; 10427.

[16] Blanco-Redondo, A., Sterke, C.M.D., Sipe, J.E., Krauss, T.F., Eggleton, B.J., Husko, C., (2016); Erratum: Pure-quartic solitons, Nature Communications 7; 11048.

[17] Biswas, A., Ullah, M.Z., Asma, M., Zhou, Q., Moshokoa, S.P., Belic, B., (2017); Optical solitons with quadratic-cubic nonlinearity by semi-inverse variational principle, Optik 
$139 ; 16-19$.

[18] Biswas, A., Triki, H., Zhou, Q., Moshokoa, S.P., Ullah, M.Z., Belic, M., (2017);Cubicquartic optical solitons in Kerr and power law media, Optik 144; 357-362.

[19] Biswas, A., Kara, A.H., Ullah, M.Z. , Zhou, Q., Triki, H., Belic, M., (2017); Conservation laws for cubic-quartic optical solitons in Kerr and power law media, Optik 145; 650-654.

[20] Bansal,A., Biswas, A., Zhou, Q. , Babatin, M.M., (2018); Lie symmetry analysis for cubic-quartic nonlinear Schrödinger's equation, Optik 169; 12-15.

[21] Biswas, A., Arshed, S., (2018); Application of semi-inverse variational principle to cubic-quartic optical solitons with Kerr and power law nonlinearity, Optik 172; 847-850.

[22] Das, A., Biswas, A., Ekici, M., Khan, S., Zhou, Q., Moshokoa, S.P., (2019); Suppressing internet bottleneck with fractional temporal evolution of cubic-quartic optical solitons, Optik 182; 303-307.

[23] Gonzalez-Gaxiola, O., Biswas, A., Mallawi, F., Belic, M., (2020); Cubic-quartic bright optical solitons by improved Adomian decomposition method, J. Adv. Res. 21;161-167.

[24] Kohl, R.W., Biswas, A., Ekici, M., Zhou, Q., Moshokoa, S.P., Belic, M.R., (2019);

Cubic-quartic optical soliton perturbation by semi-inverse variational principle, Optik 185; $45-49$.

[25] Bekir, A., Zahran, E.H.M., (2020); Bright and dark soliton solutions for the complex Kundu-Eckhaus equation; Optik; 223; 165233

[26] Bekir, A., Zahran, E.H.M., (2021); New vision for the soliton solutions to the complex Hirota-dynamical model; Phys. Scr. ; 96; 055212

[27] Kudryashov, N.A., Loguinova, N.B., (2008); Extended simplest equation method for nonlinear differential equations, Appl. Math. Comput. 205; 396-402

[28] Wazwaz, A.M., and Xu, G.Q.,(2020);Bright, dark, and Gaussons optical solutions for fourth order Schrodinger equation with cubic-quantic and logarithmic nonlinearities, Optik; $202 ; 163564$.

[29] Yildirim, Y., Biswas, A., Guggilla, P., Mallawi, F., Belic, M.R., (2020); Cubic-quartic optical solitons in birefringent fibers with four forms of nonlinear refractive index, Optik $203 ; 163885$.

[30] Yildirim, Y., Biswas, A., Jawad, A.J.M., Ekici, M., Zhou, Q., Khan, S., Alzahrani, A.K., and Belic, M.R., (2020); Cubic-quartic optical solitons in birefringent fibers with four forms of nonlinear refractive index by exp-function expansion, Results Phys.16; 102913

[31] Biswas, A., (2008); 1-Soliton solution of the $K(m ; n)$ equation with generalized evolution, Phys. Lett. A 372; 4601-4602.

[32] Triki, H., Wazwaz, A.M., (2009); Bright and dark soliton solutions for a $\mathrm{K}(\mathrm{m}$; n) equation with t-dependent coefficients, Phys. Lett. A 373; 2162-2165.

[33] Triki, H., Wazwaz, A.M., (2011); Bright and dark solitons for a generalized Kortewegde Vries-modified Korteweg-de Vries equation with high-order nonlinear terms and timedependent coefficients, Can. J. Phys. 89; 253-259. 